\title{
Monte Carlo Methods for the Ferromagnetic Potts Model Using Factor Graph Duality
}

\author{
Mehdi Molkaraie and Vicenç Gómez
}

\begin{abstract}
Normal factor graph duality offers new possibilities for Monte Carlo algorithms in graphical models. Specifically, we consider the problem of estimating the partition function of the ferromagnetic Ising and Potts models by Monte Carlo methods, which are known to work well at high temperatures, but to fail at low temperatures. We propose Monte Carlo methods (uniform sampling and importance sampling) in the dual normal factor graph, and demonstrate that they behave differently: they work particularly well at low temperatures. By comparing the relative error in estimating the partition function, we show that the proposed importance sampling algorithm significantly outperforms the state-of-the-art deterministic and Monte Carlo methods. For the ferromagnetic Ising model in an external field, we show the equivalence between the valid configurations in the dual normal factor graph and the terms that appear in the high-temperature series expansion of the partition function. Following this result, we discuss connections with JerrumSinclair's polynomial randomized approximation scheme (the subgraphs-world process) for evaluating the partition function of ferromagnetic Ising models.
\end{abstract}

Index Terms-Potts model, Ising model, normal factor graph, partition function, dual normal factor graph, Monte Carlo methods, low-temperature regime, ferromagnetism, high-temperature series expansion, subgraphs-world process.

\section{INTRODUCTION}

Many quantities of interest in statistical physics, combinatorics, information theory, and machine learning can be expressed as a partition function

$$
Z \triangleq \sum_{x_{1}, \ldots, x_{N}} f\left(x_{1}, \ldots, x_{N}\right)
$$

where $f\left(x_{1}, \ldots, x_{N}\right)$ is a nonnegative real function of finitevalued variables $x_{1}, \ldots, x_{N}$ and where the sum runs over all possible values of these variables. For example, if $f$ takes values in $\{0,1\}$, then $(1)$ counts the number of configurations $x_{1}, \ldots, x_{N}$ for which $f\left(x_{1}, \ldots, x_{N}\right) \neq 0$. In statistical physics, $Z$ is usually considered as a function of temperature (cf. Section $\mathrm{II}$ ) and hence is called the partition function. For large $N$, we are usually interested in the free energy per site $\frac{1}{N} \ln Z$ rather than in $Z$ itself.

Naive computation of (1) is exponential in $N$ and practically possible only for small $N$. If the function $f$ in 11 has a cyclefree factor graph (and no state variables with excessively many states), then the partition function can be computed exactly

M. Molkaraie is with the Department of Information Technology and Electrical Engineering, ETH Zurich, CH-8092 Zürich, Switzerland (email: mehdi.molkaraie@alumni.ethz.ch). V. Gómez is with the Artificial Intelligence and Machine Learning group at the Universitat Pompeu Fabra, 08018 Barcelona, Spain (email: vicen.gomez@upf.edu).

Parts of this work were presented in $[1]-[3]$. by sum-product message passing [4]-[6] (with complexity typically linear in $N$ ), which, in this context, coincides with the transfer matrix method from statistical physics [7, Chapter 2], [8, Chapter 5].

In general, however, the exact computation of the partition function is intractable for large $N$, and even good approximations can be hard to obtain. Empirically good approximations are often achieved with deterministic methods (including the belief propagation (BP), the generalized belief propagation (GBP) [9], and the tree expectation propagation (TreeEP) [10] algorithms), but the accuracy of such approximations is often difficult to assess theoretically.

In this paper, we pursue the idea (proposed in [1] $-[3]$ ) that normal factor graph duality offers new opportunities for Monte Carlo algorithms. We develop and test this idea for two-dimensional (2D) nearest-neighbor Ising and Potts models. Both the Ising model [11], [12] and the more general Potts model [13], [14] play an important role in many areas, including statistical physics [7], image processing [15], spatial statistics [16], and graph theory [17]-[19].

Exact computation of the partition function of the Potts model is possible only in some special cases (e.g., in the onedimensional (1D) case). For the planar Ising model without an external field, the problem is tractable and can be reduced to evaluating a certain determinant [20], [21], and this approach can also be used to obtain accurate approximations in more general settings [22]. Also there is a polynomial randomized approximation scheme [23, Chapter 28] for the partition function of general ferromagnetic Ising models in an external field due to Jerrum and Sinclair [24]. Connections among the dual normal factor graph representation of the Ising model, the approximation scheme of Jerrum and Sinclair, and the hightemperature series expansions of the partition function will be discussed in detail in this paper. However, under reasonable complexity assumptions, there is no polynomial randomized approximation scheme for the partition function of the Potts model. Indeed, for ferromagnetic Potts models, approximating the partition function is as hard as approximating the number of independent sets in a bipartite graph, which is among the presumably intractable problems [25]-[27].

Known Monte Carlo algorithms for the partition function work very well for the Potts model at high temperature (i.e., when local correlations decay quickly) [28]-[31]. At low temperatures, however, Monte Carlo methods suffer from slow and erratic convergence [30], [32]. More advanced Monte Carlo methods (e.g., nested sampling [33] and the SwendsenWang algorithm [34]) require sampling from a large sequence of constraints or intermediate distributions at different temper- 
atures to estimate the partition function. The main challenge is, therefore, to design Monte Carlo methods that achieve sufficiently fast convergence in the low-temperature regime.

The approach of this paper is based on the notions of the dual normal realization as introduced by Forney [35] and the dual normal factor graph [36]-[38]. According to the normal factor graph duality theorem, the partition function of the dual normal factor graph equals that of the primal normal factor graph up to a known scale factor [36]. The relation of normal factor graph duality to Kramers-Wannier duality [39], [14. Section II] in statistical physics has been worked out in [40][42].

Using Monte Carlo methods in the dual normal factor graph has been investigated in [1], [43], [2], [3]. It was demonstrated (by simulations) in [1] that for the $2 \mathrm{D}$ Ising model without an external field, baseline Monte Carlo methods converge faster at low temperature in the dual normal factor graph than in the primal normal factor graph. Some pertinent analytical and numerical results regarding the variance of Monte Carlo methods in the two domains were given in [43]. A suitable partitioning of variables, which allows drawing independent samples according to an auxiliary distribution, was introduced in [2] to propose an importance sampling algorithm to estimate the partition function of the Ising model in a strong external field. The methods of [2] were further generalized in [3] to models with a mixture of strong and weak couplings.

In this paper, we further explore the use of Monte Carlo methods in the dual normal factor graph. Specifically, we propose Monte Carlo methods for estimating the partition function of ferromagnetic $q$-state Potts models, with or without an external field. We consider uniform sampling and importance sampling algorithms, both of which are shown to work very well for strong couplings or, equivalently, at low temperature.

Our experimental results show that, in various settings, the importance sampling algorithm significantly improves upon the state-of-the-art Monte Carlo and deterministic methods. Indeed, in contrast to Monte Carlo methods in the primal domain, the dual-domain Monte Carlo algorithms of this paper excel at low temperatures.

The paper is organized as follows. In Section $\Pi$, we review the Potts model. The primal and the dual normal factor graphs of the model will be presented in Section [III and Section IV. respectively. Specific Monte Carlo algorithms that use the dual normal factor graph are proposed in Section $\mathrm{V}$, and pertinent experimental results (including comparisons with standard deterministic and Monte Carlo methods) are presented in Section VI. Extensions of our Monte Carlo methods to the Potts model in an external field are discussed in Section VII In Section VIII we establish the connection among the valid configurations in the dual normal factor graph representation of the Ising model in an external field, high temperature series expansions of the partition function, and the randomized approximation scheme of Jerrum and Sinclair. Appendix A compares the variance of Monte Carlo methods in the primal and in the dual normal factor graphs of the 2D Ising model to demonstrate their opposite behavior.

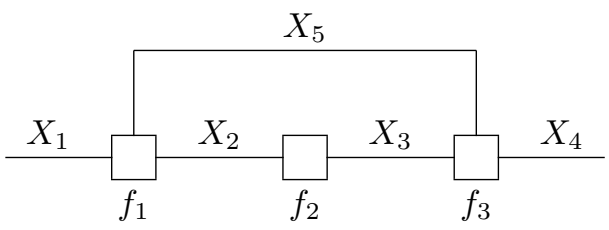

Fig. 1: Normal factor graph of 10 .

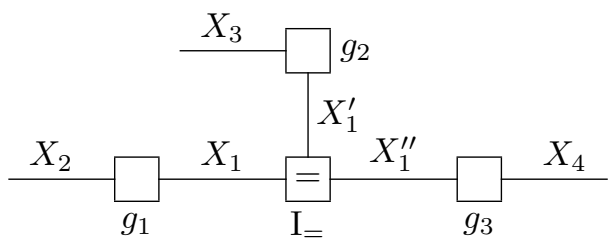

Fig. 2: Normal factor graph of 11 .

\section{The 2D Potts Model without External Field}

Let $X_{1}, X_{2}, \ldots, X_{N}$ be a collection of $N$ random variables that take values in the set $\mathcal{X}$, which in this context is identical to $\mathbb{Z} / q \mathbb{Z}$, the ring of integers modulo $q$ for some fixed integer $q \geq 2$. (In the special case where $q=2$, we obtain the Ising model.) Let $x_{i}$ represent a possible realization of $X_{i}$ and $\mathbf{X}$ stand for $\left(X_{1}, X_{2}, \ldots, X_{N}\right)$. The vectors $\mathbf{x} \in \mathcal{X}^{N}$ will be called configurations.

The variables $X_{1}, X_{2}, \ldots, X_{N}$ are associated with the vertices of a simple and connected graph $\mathcal{G}=(\mathcal{V}, \mathcal{E})$ that has $N=|\mathcal{V}|$ vertices and $|\mathcal{E}|$ edges. In the Potts model, each variable represents the $q$ possible states of a particle, and two variables interact if their corresponding vertices are connected by an edge in $\mathcal{E}$.

For illustrative purposes, we will take $\mathcal{G}$ to be a grid with size $N=M \times M$, and assume periodic boundary conditions, so that each variable has exactly four neighbors. However, the methods of this paper are easily adapted to Potts models with arbitrary topology. Indeed, we will consider 2D Potts models with free boundary conditions in our numerical experiments.

Conventionally $\mathcal{E}$ is defined as the set of all the unordered pairs $(k, \ell) \in\{1, \ldots, N\} \times\{1, \ldots, N\}$ such that $X_{k}$ and $X_{\ell}$ are nearest neighbors. Thus

$$
|\mathcal{E}|=2 N
$$

for periodic boundary conditions. A real coupling parameter $J_{k, \ell}$ is associated with each pair $(k, \ell) \in \mathcal{E}$. The energy of a configuration $\mathrm{x} \in \mathcal{X}^{N}$ is given by the Hamiltonian

$$
\mathcal{H}(\mathbf{x})=-\sum_{(k, \ell) \in \mathcal{E}} J_{k, \ell} \cdot \delta\left(x_{k}-x_{\ell}\right),
$$

where $\delta(\cdot)$ is the Kronecker delta, which evaluates to one if its argument is zero, and to zero otherwise.

In this paper, we focus on ferromagnetic models, which are characterized by the condition $J_{k, \ell} \geq 0$ for all $(k, \ell) \in \mathcal{E}$, i.e., configurations in which adjacent variables take on the same value have lower energy. The probability of a configuration $\mathrm{x} \in \mathcal{X}^{N}$ is given by the Boltzmann distribution

$$
p_{\mathrm{B}}(\mathbf{x})=\frac{e^{-\beta \mathcal{H}(\mathbf{x})}}{Z} \text {. }
$$


Here, $\beta$ denotes the inverse temperature and the normalization constant $Z$ is the partition function given by

$$
Z=\sum_{\mathbf{x} \in \mathcal{X}^{N}} e^{-\beta \mathcal{H}(\mathbf{x})}
$$

where the sum runs over all the configurations [7].

We will find it convenient to omit the parameter $\beta$ (i.e., we set $\beta=1$ ) and to work with varying values of the coupling parameters $J_{k, \ell}$. In this set-up, large values of $J_{k, \ell}$ correspond to low temperature and small values correspond to high temperature. In particular, the special case where $J_{k, \ell}=0$ for all $(k, \ell) \in \mathcal{E}$ corresponds to infinite temperature.

We now let

$$
\begin{aligned}
f(\mathbf{x}) & =e^{-\mathcal{H}(\mathbf{x})} \\
& =\prod_{(k, \ell) \in \mathcal{E}} \kappa_{k, \ell}\left(x_{k}, x_{\ell}\right)
\end{aligned}
$$

with

$$
\kappa_{k, \ell}\left(x_{k}, x_{\ell}\right)= \begin{cases}e^{J_{k, \ell},} & \text { if } x_{k}=x_{\ell} \\ 1, & \text { otherwise. }\end{cases}
$$

Thus (5) becomes

$$
Z=\sum_{\mathbf{x} \in \mathcal{X}^{N}} f(\mathbf{x})
$$

in agreement with (1).

The factorization (7) will be used in the next section.

\section{Primal Normal FACtOR Graph OF THE POTTS MODEL}

We use normal factor graphs as in [35], [5], [36], [37] (also called Forney factor graphs), where variables are represented by edges and factors are represented by nodes/boxes. (By contrast, factor graphs as in [4] represent both factors and variables by nodes.) For example, the factorization

$$
\begin{aligned}
& f\left(x_{1}, x_{2}, x_{3}, x_{4}, x_{5}\right) \\
& \quad=f_{1}\left(x_{1}, x_{2}, x_{5}\right) f_{2}\left(x_{2}, x_{3}\right) f_{3}\left(x_{3}, x_{4}, x_{5}\right)
\end{aligned}
$$

is represented by the normal factor graph in Fig. 1 . We say that a configuration $\mathbf{x}$ is valid iff $f(\mathbf{x}) \neq 0$, and we note that only valid configurations contribute to the partition function (1).

As observed in [35], in order to represent the variables by edges, each variable must be involved in only one or two factors. If some variable appears in more than two factors as, e.g., in

$$
g\left(x_{1}, x_{2}, x_{3}, x_{4}\right)=g_{1}\left(x_{1}, x_{2}\right) g_{2}\left(x_{1}, x_{3}\right) g_{3}\left(x_{1}, x_{4}\right),
$$

we introduce auxiliary variables $x_{1}^{\prime}$ and $x_{1}^{\prime \prime}$, and define an additional equality indicator function

$$
\mathrm{I}_{=}\left(x_{1}, x_{1}^{\prime}, x_{1}^{\prime \prime}\right) \triangleq \delta\left(x_{1}-x_{1}^{\prime}\right) \cdot \delta\left(x_{1}-x_{1}^{\prime \prime}\right)
$$

(as shown in Fig. 2) such that $x_{1}=x_{1}^{\prime}=x_{1}^{\prime \prime}$ in all valid configurations. The partition function is not affected by such replications.

We next note that the Hamiltonian (3) and factors (8) can equivalently be written as a function of $y_{k, \ell}=x_{k}-x_{\ell}$. To

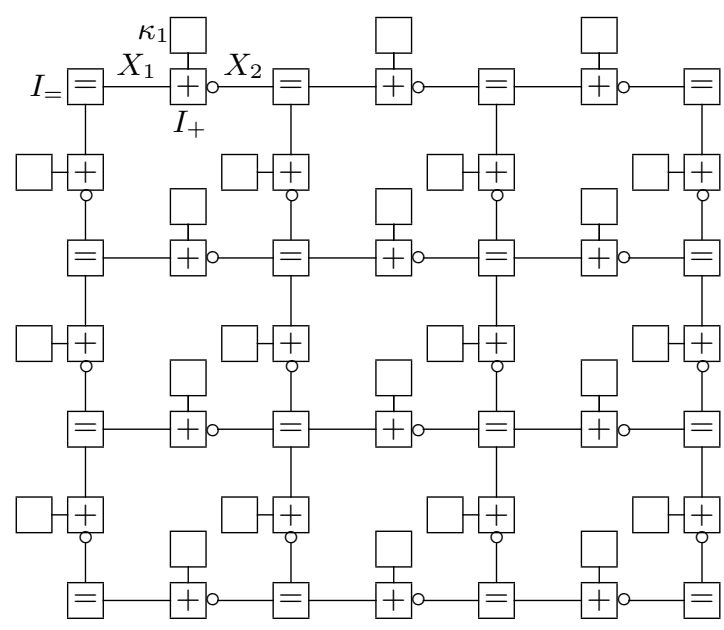

Fig. 3: Primal normal factor graph of the 2D Potts model. The empty boxes represent the factors $(8)$, the boxes labeled "=" are equality indicator functions given by (12), the boxes labeled "+" are zero-sum indicator functions given by (14), and the symbol "o" indicates a sign inversion. The periodic boundary conditions are not shown.

simplify notation, we will henceforth denote the elements of $\mathcal{E}$ by a single index variable $e \in \mathcal{E}$ rather than by a vertex index pair $(k, \ell) \in \mathcal{V}^{2}$, with adjacencies continuing to be determined by the graph $\mathcal{G}=(\mathcal{V}, \mathcal{E})$.

Hence, each factor (8) can be written as

$$
\kappa_{e}\left(y_{e}\right)= \begin{cases}e^{J_{e}}, & \text { if } y_{e}=0 \\ 1, & \text { otherwise. }\end{cases}
$$

Applying factors (13) in (7), we can construct the primal normal factor graph of the Potts model as shown in Fig. 3 in which the empty boxes represent (13), the boxes labeled "=" are instances of equality indicator functions as in (12), the boxes labeled "+" are instances of the zero-sum indicator functions defined as

$$
\mathrm{I}_{+}\left(y_{e}, x_{k}, x_{\ell}\right) \triangleq \delta\left(y_{e}+x_{k}+x_{\ell}\right),
$$

and in analogy with the logic NAND gate the symbol "o" is used to indicate a sign inversion. (Recall that all arithmetic manipulations are done modulo $q$.)

For $q=2$, the Potts model is equivalent to the Ising model. However, the standard convention is to define the Hamiltonian of the model as

$$
\mathcal{H}(\mathbf{x})=-\sum_{(k, \ell) \in \mathcal{E}} J_{k, \ell} \cdot\left(2 \delta\left(x_{k}-x_{\ell}\right)-1\right) .
$$

Following a similar approach, we can obtain the primal normal factor graph of the Ising model (also shown in Fig. 3), where the empty boxes represent factors given by

$$
\kappa_{e}\left(y_{e}\right)= \begin{cases}e^{J_{e}}, & \text { if } y_{e}=0 \\ e^{-J_{e}}, & \text { if } y_{e}=1\end{cases}
$$

Note that, for the Ising model the "o" symbols are immaterial and can be removed from Fig. 3 . 


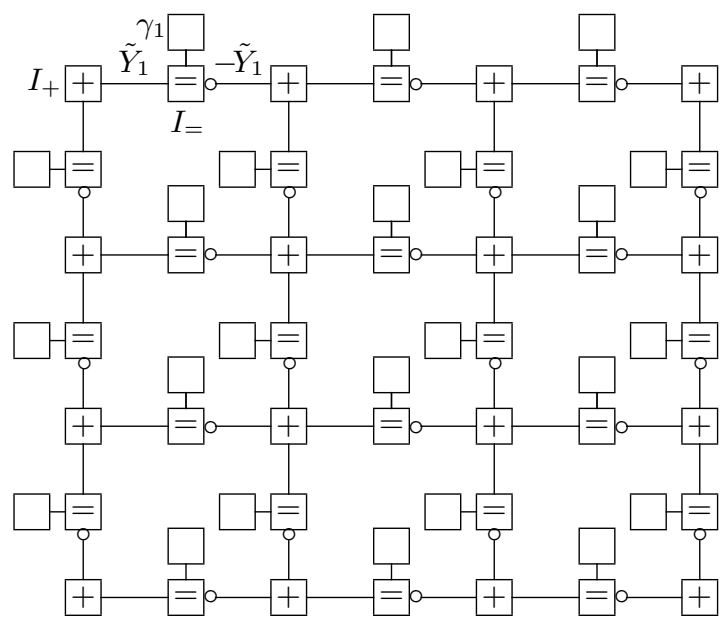

Fig. 4: Dual normal factor graph of the 2D Potts model. The empty boxes represent the factors $(18)$, the boxes labeled "=" are equality indicator functions given by (12), the boxes labeled "+" are zero-sum indicator functions given by (14), and the symbol "o" indicates a sign inversion. The periodic boundary conditions are not shown.

In the special case of the 2D Ising model with constant couplings $J_{e}=J$ and without an external field, the partition function is analytically available in the thermodynamic limit (i.e., as $N \rightarrow \infty$ ) from Onsager's solution [44]. In Appendix A we will use the analytical solution of the partition function to analyze the variance of Monte Carlo methods of this paper. For the (nonbinary) 2D Potts model, no such analytical solution for the partition function is yet available.

Next, we will describe the corresponding dual normal factor graphs of the models in this section.

\section{Dual Normal Factor GRAPH OF THE POTTS MODEL}

The dual normal factor graph of some given (primal) normal factor graph has the same topology as the primal normal factor graph, but all factors are replaced by their Fourier transforms (which includes replacing equality indicator functions by zerosum indicator functions, and vice versa). See [35], [5], [36], [38], [43] for more details.

In the dual normal factor graph, all variables are replaced by their corresponding dual (frequency) variables, which take values in the same alphabet as the primal variables. We will use the tilde symbol to denote variables in the dual domain. The dual normal factor graph has the same partition function as the primal normal factor graph, up to some known scale factor [36, Theorem 2]. We denote the partition function of the dual normal factor graph by $Z_{\mathrm{d}}$.

Following [43], we can obtain the dual normal factor graph of the 2D Potts model as shown in Fig. 4, in which the empty boxes represent factors that are the 1D Fourier transforms of (13) given by

$$
\gamma_{e}\left(\tilde{y}_{e}\right)=\sum_{y_{e} \in \mathcal{X}} \kappa_{e}\left(y_{e}\right) e^{-\mathrm{i} 2 \pi y_{e} \tilde{y}_{e} / q} .
$$

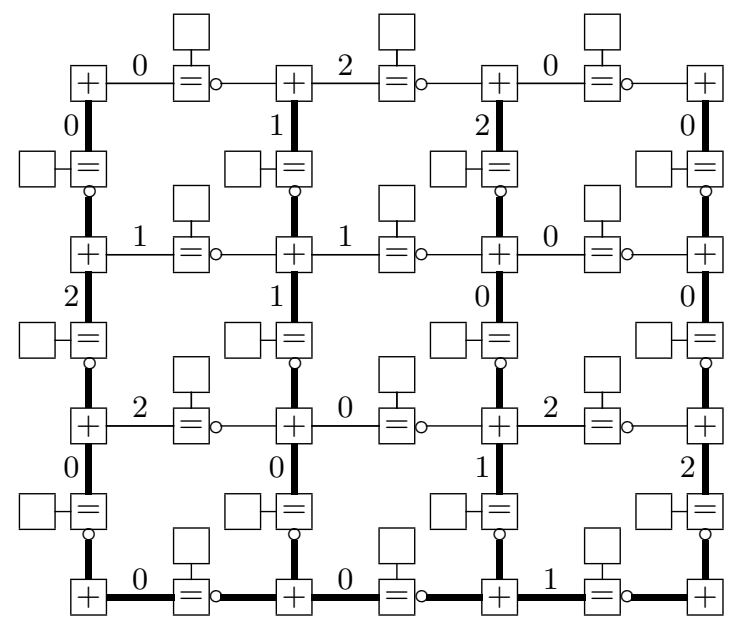

Fig. 5: A partitioning of the variables $\tilde{\mathbf{Y}}=\left\{\tilde{Y}_{e}: e \in \mathcal{E}\right\}$ in Fig. 4 as in Section IV-A The edges in $\mathcal{T}$ (drawn with thick edges) represent the variables $\tilde{\mathbf{Y}}_{\mathcal{T}}$, which are linearly dependent on the remaining variables $\tilde{\mathbf{Y}}_{\overline{\mathcal{T}}}$. This spanning tree works both with and without periodic boundary conditions. Also shown is an example of a valid configuration for $q=3$ (assuming no boundary conditions).

Thus

$$
\gamma_{e}\left(\tilde{y}_{e}\right)= \begin{cases}e^{J_{e}}-1+q, & \text { if } \tilde{y}_{e}=0 \\ e^{J_{e}}-1, & \text { otherwise, }\end{cases}
$$

which is nonnegative due to the ferromagnetic assumption (i.e., $J_{e} \geq 0$ )

Similarly, we can obtain the dual normal factor graph of the 2D Ising model (shown in Fig. 4), where the empty boxes represent factors as in

$$
\gamma_{e}\left(\tilde{y}_{e}\right)= \begin{cases}2 \cosh \left(J_{e}\right), & \text { if } \tilde{y}_{e}=0 \\ 2 \sinh \left(J_{e}\right), & \text { if } \tilde{y}_{e}=1,\end{cases}
$$

which is the 1D Fourier transform of (16). Notice that (19) is also nonnegative due to ferromagnetic assumption. Again, for the Ising model the "o" symbols can be safely removed from the dual normal factor graph.

The partition function of the dual normal factor graph is thus

$$
Z_{\mathrm{d}}=\sum_{\text {valid } \tilde{\mathbf{y}}} \prod_{e \in \mathcal{E}} \gamma_{e}\left(\tilde{y}_{e}\right)
$$

where the sum runs over all valid configurations in the dual normal factor graph.

For the dual 2D Potts and Ising models with factors as in (18) and (19), and with periodic boundary conditions, it holds that

$$
Z_{\mathrm{d}}=q^{N} Z
$$

see [36].

\footnotetext{
${ }^{1}$ In general, the scale factor $\alpha(\mathcal{G})=Z_{\mathrm{d}} / Z$ depends on the topology of $\mathcal{G}$ and on the local scale factors used in the Fourier transforms. In our setup, the scale factor is given by $\alpha(\mathcal{G})=q^{|\mathcal{E}|-|\mathcal{V}|}$. For example, in a 2D torus $|\mathcal{E}|=2 N$, and therefore $\alpha(\mathcal{G})=q^{N}$ as in 21 ; in a 1D model with periodic boundary conditions $|\mathcal{E}|=|\mathcal{V}|$, and thus $\alpha(\mathcal{G})=1$. For more details, see [45], $[42$ Section 3.3].
} 
Fig. 4 is the basis of the Monte Carlo algorithms of this paper to estimate $Z_{\mathrm{d}}$. The estimates are then used to compute an estimate of $Z$ via 21 .

\section{A. Independent Variables and Spanning Trees in the Dual Normal Factor Graph}

In our Monte Carlo methods, we will use partitions of $\mathcal{G}$ into two disjoint subsets $\mathcal{G}=\mathcal{T} \cup \overline{\mathcal{T}}$ such that $\mathcal{T}$ is a spanning tree (that reaches every zero-sum factor) and $\overline{\mathcal{T}}$ is the corresponding cospanning tree, as illustrated in Fig. 5 . The edges of $\mathcal{T}$ are called the branches and the edges of $\mathcal{T}$ are called the chords of $\mathcal{G}$ with respect to $\mathcal{T}$. Although $\mathcal{T}$ is always without cycles, $\overline{\mathcal{T}}$ need not be cycle-free. Any such partition induces a corresponding partition of the variables $\tilde{\mathbf{Y}}=\left\{\tilde{Y}_{e}: e \in \mathcal{E}\right\}$ into $\tilde{\mathbf{Y}}_{\overline{\mathcal{T}}}$ and $\tilde{\mathbf{Y}}_{\mathcal{T}}$.

Proposition 1. Consider a valid configuration in the dual normal factor graph of the Potts model. Suppose variables $\tilde{Y}_{1}, \tilde{Y}_{2}, \ldots$ form a cutset in the dual normal factor graph, then it holds that

$$
\sum_{e \in \text { Cutset }} \tilde{y}_{e}=0
$$

Proof. Removing all the edges that represent variables $\tilde{Y}_{1}, \tilde{Y}_{2}, \ldots$ partitions $\mathcal{G}$ into $\mathcal{G}_{1} \cup \mathcal{G}_{2}$. Suppose in $\mathcal{G}_{1}$ we write down the equations associated with all the zero-sum indicator factors. Since each variable, say $\tilde{y}_{t}$ for $t \in \mathcal{E}_{1}$ appears twice in the summation, once as $\tilde{y}_{t}$ and once as $-\tilde{y}_{t}$ (see Fig. 4), the sum over all these equations is equal to zero. Furthermore, the same sum in $\mathcal{G}$ is equal to $\sum_{e \in \text { Cutset }} \tilde{y}_{e}$. This completes the proof.

The proof follows along the same lines in $\mathcal{G}_{2}$. For more details, see [45], [42, Section 2.5].

Removing a branch $b \in \mathcal{T}$ partitions $\mathcal{T}=\mathcal{T}_{1} \cup \mathcal{T}_{2}$. The edges that connect $\mathcal{T}_{1}$ and $\mathcal{T}_{2}$ form a unique cutset in $\mathcal{G}$, called the fundamental cutset belonging to $b$. Each fundamental cutset has exactly one branch of $\mathcal{T}$ that does not appear in any other fundamental cutset, along with edges (chords) that belong to $\overline{\mathcal{T}}$. Indeed, each spanning tree defines a set of $|\mathcal{T}|$ fundamental cutsets: one for each branch of the spanning tree [46, Chapter 2]. According to Proposition 1. for each $b \in \mathcal{T}$ we can compute $\tilde{Y}_{b}$ as a linear combination of $\tilde{\mathbf{Y}}_{\overline{\mathcal{T}}}$ by applying 22 . on the fundamental cutset belonging to $b$. We conclude that the variables in $\tilde{\mathbf{Y}}_{\overline{\mathcal{T}}}$ are linearly independent and the variables in $\tilde{\mathbf{Y}}_{\mathcal{T}}$ are fully determined by $\tilde{\mathbf{Y}}_{\overline{\mathcal{T}}}$ via a linear transformation.

It follows that the number of valid configurations in the dual normal factor graph of the Potts model is $q^{|\overline{\mathcal{T}}|}$. In any such partitioning the number of variables in $\tilde{\mathbf{Y}}_{\mathcal{T}}$ is

$$
|\mathcal{T}|=N-1
$$

and the number of variables in $\tilde{\mathbf{Y}}_{\overline{\mathcal{T}}}$ is $|\overline{\mathcal{T}}|=|\mathcal{E}|-|\mathcal{T}|$. For the $2 \mathrm{D}$ torus, we thus have

$$
|\overline{\mathcal{T}}|=N+1
$$

from (2).

\section{Monte Carlo Methods for the Partition FUnCTION OF THE DUAL NORMAL FACTOR GRAPH}

We propose two basic Monte Carlo algorithms for estimating the partition function. Both algorithms use partitions of $\mathcal{E}$ and $\mathbf{Y}$ as in Section IV-A and Fig. 5.

In both Monte Carlo algorithms, we draw independent samples $\tilde{\mathbf{y}}_{\overline{\mathcal{T}}}^{(1)}, \ldots, \tilde{\mathbf{y}}_{\overline{\mathcal{T}}}^{(L)} \in \mathcal{X}^{|\overline{\mathcal{T}}|}$ according to some auxiliary probability distribution, and each of these samples $\tilde{\mathbf{y}}_{\overline{\mathcal{T}}}^{(\ell)}$ is completed (by computing the corresponding $\tilde{\mathbf{y}}_{\mathcal{T}}^{(\ell)} \in \mathcal{X}^{|\mathcal{T}|}$ ) to a valid configuration $\tilde{\mathbf{y}}^{(\ell)}=\left(\tilde{\mathbf{y}}_{\overline{\mathcal{T}}}^{(\ell)}, \tilde{\mathbf{y}}_{\mathcal{T}}^{(\ell)}\right) \in \mathcal{X}^{|\mathcal{E}|}$. Computing $\tilde{\mathbf{y}}_{\mathcal{T}}^{(\ell)}$ from $\tilde{\mathbf{y}}_{\overline{\mathcal{T}}}^{(\ell)}$ is easy and linear in $|\mathcal{T}|$.

We will also use the quantities

$$
\begin{aligned}
\Gamma_{\overline{\mathcal{T}}}\left(\tilde{\mathbf{y}}_{\mathcal{\mathcal { T }}}\right) & =\prod_{e \in \overline{\mathcal{T}}} \gamma_{e}\left(\tilde{y}_{e}\right), \\
\Gamma_{\mathcal{T}}\left(\tilde{\mathbf{y}}_{\mathcal{T}}\right) & =\prod_{e \in \mathcal{T}} \gamma_{e}\left(\tilde{y}_{e}\right),
\end{aligned}
$$

and

$$
\Gamma(\tilde{\mathbf{y}})=\Gamma_{\overline{\mathcal{T}}}\left(\tilde{\mathbf{y}}_{\overline{\mathcal{T}}}\right) \Gamma_{\mathcal{T}}\left(\tilde{\mathbf{y}}_{\mathcal{T}}\right)=\prod_{e \in \mathcal{E}} \gamma_{e}\left(\tilde{y}_{e}\right),
$$

therefore (20) becomes

$$
Z_{\mathrm{d}}=\sum_{\text {valid } \tilde{\mathbf{y}}} \Gamma(\tilde{\mathbf{y}})
$$

We propose uniform sampling and importance sampling algorithms to estimate $Z_{\mathrm{d}}$. Given the partitioning, the computational complexity of our algorithms is $O(|\mathcal{E}|)$ per sample and $O(L|\mathcal{E}|)$ in total. The variance of both methods is derived in Section $\mathrm{V}-\mathrm{C}$.

\section{A. Uniform Sampling}

As a baseline algorithm (used in [1] and [43]), we use independent samples $\tilde{\mathbf{y}}_{\overline{\mathcal{T}}}^{(1)}, \ldots, \tilde{\mathbf{y}}_{\overline{\mathcal{T}}}^{(L)}$ drawn uniformly over $\mathcal{X}^{|\overline{\mathcal{T}}|}$, which are completed to valid configurations $\tilde{\mathbf{y}}^{(\ell)} \in \mathcal{X}^{|\mathcal{E}|}$ as described above. We then use the estimate

$$
\hat{Z}_{\mathrm{d}}^{\mathrm{Uni}}=\frac{q^{|\overline{\mathcal{T}}|}}{L} \sum_{\ell=1}^{L} \Gamma\left(\tilde{\mathbf{y}}^{(\ell)}\right) .
$$

It is easily verified that $\mathrm{E}\left[\hat{Z}_{\mathrm{d}}^{\mathrm{Uni}}\right]=Z_{\mathrm{d}}$, i.e., the estimator is unbiased:

$$
\begin{aligned}
\mathrm{E}\left[\frac{q^{|\overline{\mathcal{T}}|}}{L} \sum_{\ell=1}^{L} \Gamma\left(\tilde{\mathbf{Y}}^{(\ell)}\right)\right] & =q^{|\overline{\mathcal{T}}|} \mathrm{E}\left[\Gamma\left(\tilde{\mathbf{Y}}^{(1)}\right)\right] \\
& =q^{|\overline{\mathcal{T}}|} \sum_{\text {valid } \tilde{\mathbf{y}}} \frac{1}{q^{|\overline{\mathcal{T}}|}} \Gamma(\tilde{\mathbf{y}}) \\
& =Z_{\mathrm{d}},
\end{aligned}
$$

where the last step follows from 28.

The accuracy of 29p depends on the fluctuations of $\Gamma\left(\tilde{\mathbf{y}}^{(\ell)}\right)$. In the low-temperature limit (i.e., for $e^{J_{e}} \gg q$ ), these fluctuations disappear, because $\gamma_{e}\left(\tilde{y}_{e}\right) \approx e^{J_{e}}$ becomes constant. The estimator (29) can therefore be expected to work well at sufficiently low temperatures. 


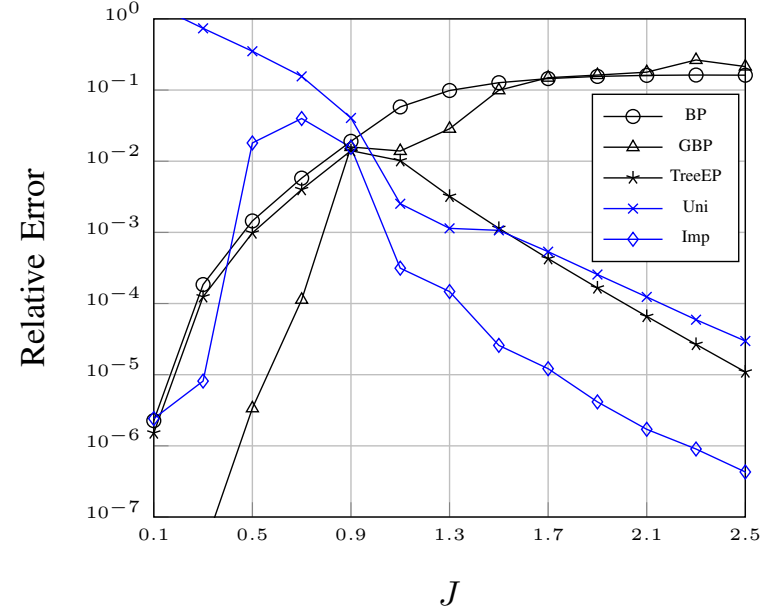

Fig. 6: Comparison with deterministic algorithms (BP, GBP, and TreeEP): experimental results for a Potts model with $q=3, N=8 \times 8$, periodic boundary conditions, and constant couplings $J$. The plot shows the relative error (51) as a function of $J$. (Recall that large $J$ corresponds to the lowtemperature regime.)

\section{B. Importance Sampling}

An importance sampling estimator (proposed in [2], [3]) is obtained by drawing independent samples $\tilde{\mathbf{y}}_{\overline{\mathcal{T}}}^{(1)}, \ldots, \tilde{\mathbf{y}}_{\overline{\mathcal{T}}}^{(L)} \in$ $\mathcal{X}^{|\overline{\mathcal{T}}|}$ according to the auxiliary probability distribution

$$
\begin{aligned}
p_{\overline{\mathcal{T}}}\left(\tilde{\mathbf{y}}_{\overline{\mathcal{T}}}\right) & =\frac{\Gamma_{\overline{\mathcal{T}}}\left(\tilde{\mathbf{y}}_{\overline{\mathcal{T}}}\right)}{Z_{\overline{\mathcal{T}}}} \\
& =\prod_{e \in \overline{\mathcal{T}}} \frac{\gamma_{e}\left(\tilde{y}_{e}\right)}{\sum_{\xi=0}^{q-1} \gamma_{e}(\xi)},
\end{aligned}
$$

where $Z_{\overline{\mathcal{T}}}$ is available in closed-form as

$$
\begin{aligned}
Z_{\overline{\mathcal{T}}} & =\prod_{e \in \overline{\mathcal{T}}} \sum_{\xi=0}^{q-1} \gamma_{e}(\xi) \\
& =\prod_{e \in \overline{\mathcal{T}}} q e^{J_{e}} \\
& =q^{|\overline{\mathcal{T}}|} \exp \left(\sum_{e \in \overline{\mathcal{T}}} J_{e}\right) .
\end{aligned}
$$

The product form in 34 indicates that to draw samples according to $p_{\overline{\mathcal{T}}}\left(\tilde{\mathbf{y}}_{\overline{\mathcal{T}}}\right)$, we can draw each component $\tilde{y}_{e}^{(\ell)}$ of $\tilde{\mathbf{y}}_{\overline{\mathcal{T}}}^{(\ell)}$ independently with probability

$$
\begin{aligned}
& P\left(\tilde{y}_{e}^{(\ell)}=\xi\right) \\
& \quad= \begin{cases}\frac{1+(q-1) e^{-J_{e}}}{q}, & \text { if } \xi=0 \\
\frac{1-e^{-J_{e}}}{q}, & \text { for } \xi=1,2, \ldots, q-1 .\end{cases}
\end{aligned}
$$

Again, the samples $\tilde{\mathbf{y}}_{\overline{\mathcal{T}}}^{(1)}, \ldots, \tilde{\mathbf{y}}_{\overline{\mathcal{T}}}^{(L)}$ are completed to valid configurations $\tilde{\mathbf{y}}^{(1)}, \ldots, \tilde{\mathbf{y}}^{(L)} \in \mathcal{X}^{|\mathcal{E}|}$. We then use the estimate

$$
\hat{Z}_{\mathrm{d}}^{\mathrm{Imp}}=\frac{Z_{\overline{\mathcal{T}}}}{L} \sum_{\ell=1}^{L} \Gamma_{\mathcal{T}}\left(\tilde{\mathbf{y}}_{\mathcal{T}}^{(\ell)}\right),
$$

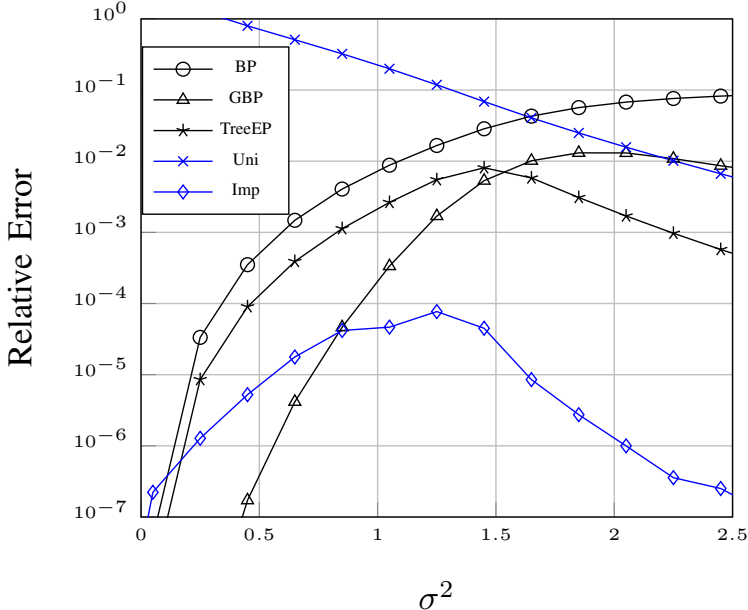

Fig. 7: Comparison with deterministic algorithms (BP, GBP, and TreeEP): experimental results for a Potts model with $q=3, N=8 \times 8$, periodic boundary conditions, and coupling parameters $J_{e}: J_{e}=\left|J_{e}^{\prime}\right|$ with $J_{e}^{\prime} \stackrel{\text { i.i.d. }}{\sim} \mathcal{N}\left(0, \sigma^{2}\right)$. The plot shows the relative error 51) as a function of $\sigma^{2}$.

which is unbiased:

$$
\begin{aligned}
\mathrm{E}\left[\frac{Z_{\overline{\mathcal{T}}}}{L} \sum_{\ell=1}^{L} \Gamma_{\mathcal{T}}\left(\tilde{\mathbf{Y}}_{\mathcal{T}}^{(\ell)}\right)\right] & =Z_{\overline{\mathcal{T}}} \mathrm{E}\left[\Gamma_{\mathcal{T}}\left(\tilde{\mathbf{Y}}_{\mathcal{T}}^{(1)}\right)\right] \\
& =Z_{\overline{\mathcal{T}}} \sum_{\text {valid } \tilde{\mathbf{y}}} p_{\overline{\mathcal{T}}}\left(\tilde{\mathbf{y}}_{\overline{\mathcal{T}}}\right) \Gamma_{\mathcal{T}}\left(\tilde{\mathbf{y}}_{\mathcal{T}}\right) \\
& =\sum_{\text {valid } \tilde{\mathbf{y}}} \Gamma_{\overline{\mathcal{T}}}\left(\tilde{\mathbf{y}}_{\overline{\mathcal{T}}}\right) \Gamma_{\mathcal{T}}\left(\tilde{\mathbf{y}}_{\mathcal{T}}\right) \\
& =Z_{\text {d }}
\end{aligned}
$$

The accuracy of (39) mainly depends on the fluctuations of $\Gamma_{\mathcal{T}}\left(\tilde{\mathbf{y}}_{\mathcal{T}}^{(\ell)}\right)$. The estimator can therefore be expected to work well at sufficiently low temperatures where these fluctuations disappear.

\section{Variance of the Estimates}

The variance of the importance sampling estimator (39) is

$$
\begin{aligned}
& \operatorname{Var}\left[\hat{Z}_{\mathrm{d}}^{\mathrm{Imp}}\right]=\mathrm{E}\left[\left(\hat{Z}_{\mathrm{d}}^{\mathrm{Imp}}\right)^{2}\right]-\mathrm{E}\left[\hat{Z}_{\mathrm{d}}^{\mathrm{Imp}}\right]^{2} \\
& =\frac{Z_{\overline{\mathcal{T}}}^{2}}{L}\left(\sum_{\text {valid } \tilde{\mathbf{y}}} p_{\overline{\mathcal{T}}}\left(\tilde{\mathbf{y}}_{\overline{\mathcal{T}}}\right) \Gamma_{\mathcal{T}}\left(\tilde{\mathbf{y}}_{\mathcal{T}}\right)^{2}\right)-\frac{Z_{\mathrm{d}}^{2}}{L} \\
& =\frac{1}{L}\left(\sum_{\text {valid } \tilde{\mathbf{y}}} \frac{\Gamma_{\overline{\mathcal{T}}}\left(\tilde{\mathbf{y}}_{\overline{\mathcal{T}}}\right)^{2}}{p_{\overline{\mathcal{T}}}\left(\tilde{\mathbf{y}}_{\overline{\mathcal{T}}}\right)} \Gamma_{\mathcal{T}}\left(\tilde{\mathbf{y}}_{\mathcal{T}}\right)^{2}\right)-\frac{Z_{\mathrm{d}}^{2}}{L} \\
& =\frac{Z_{\mathrm{d}}^{2}}{L}\left(\sum_{\text {valid } \tilde{\mathbf{y}}} \frac{p_{\mathrm{d}}(\tilde{\mathbf{y}})^{2}}{p_{\tilde{\mathcal{T}}}(\tilde{\mathbf{y}})}-1\right),
\end{aligned}
$$

where both $p_{\mathrm{d}}(\tilde{\mathbf{y}})=\Gamma(\tilde{\mathbf{y}}) / Z_{\mathrm{d}}$ and $p_{\overline{\mathcal{T}}}\left(\tilde{\mathbf{y}}_{\overline{\mathcal{T}}}\right)=\Gamma_{\overline{\mathcal{T}}}\left(\tilde{\mathbf{y}}_{\overline{\mathcal{T}}}\right) / Z_{\overline{\mathcal{T}}}$ are probability mass functions defined on the valid configurations of the dual normal factor graph.

We thus have

$$
\operatorname{Var}\left[\hat{Z}_{\mathrm{d}}^{\mathrm{Imp}}\right] \frac{L}{Z_{\mathrm{d}}^{2}}=\chi^{2}\left(p_{\mathrm{d}}, p_{\overline{\mathcal{T}}}\right),
$$


where $\chi^{2}(\cdot, \cdot)$ denotes the chi-squared divergence, which is always nonnegative and is equal to zero if and only if its two arguments are equal [47, Chapter 4].

An analogous derivation for the uniform sampling estimator (29) yields

$$
\operatorname{Var}\left[\hat{Z}_{\mathrm{d}}^{\mathrm{Uni}}\right] \frac{L}{Z_{\mathrm{d}}^{2}}=\chi^{2}\left(p_{\mathrm{d}}, p_{\mathrm{u}}\right),
$$

where $p_{\mathrm{u}}(\tilde{\mathbf{y}})$ is the uniform distribution over the valid configurations.

In the low-temperature limit with $e^{J_{e}} \gg q$ for all $e \in \mathcal{E}$, both $p_{\mathrm{d}}$ and $p_{\overline{\mathcal{T}}}$ become uniform over the valid configurations and both (48) and (49) vanish. More importantly, the variance of the importance sampling estimator (48) vanishes under the weaker condition (weaker for nonconstant couplings)

$$
e^{J_{e}} \gg q \text { for } e \in \mathcal{T},
$$

since in this case $p_{\mathrm{d}}\left(\tilde{\mathbf{y}}_{\mathbf{\mathbf { y }}} \propto \Gamma_{\overline{\mathcal{T}}}\left(\tilde{\mathbf{y}}_{\overline{\mathcal{T}}}\right) \Gamma_{\mathcal{T}}\left(\tilde{\mathbf{y}}_{\mathcal{T}}\right)\right.$ converges to $p_{\overline{\mathcal{T}}}(\tilde{\mathbf{y}}) \propto \Gamma_{\overline{\mathcal{T}}}\left(\tilde{\mathbf{y}}_{\overline{\mathcal{T}}}\right)$ if $\Gamma_{\mathcal{T}}\left(\tilde{\mathbf{y}}_{\mathcal{T}}\right)$ becomes constant.

For the Ising model on a 2D torus, a more detailed analysis of the variance of our proposed Monte Carlo methods is given in Appendix A

\section{Choosing the Partitioning}

The choice of $\mathcal{T}$ and $\overline{\mathcal{T}}$ does not affect the performance of the uniform sampling estimator (29), but it can affect the performance (i.e., the convergence) of the importance sampling estimator (39) for nonconstant couplings. Recall that the normalized variance (48) vanishes if (50) holds. This result suggests to include into $\mathcal{T}$ only edges $e$ with stronger couplings (i.e., with large $J_{e}$ ). To this end, the following heuristic strategy can be used: choose $\mathcal{T}$ to be a spanning tree that maximizes $\sum_{e \in \mathcal{T}} J_{e}$. This is a maximum-spanning tree problem, that can be solved efficiently with complexity linear in $N$ and $|\mathcal{E}|$ (see [48, Chapter. VI]). We will use this heuristic strategy in our numerical experiments in Section VI

\section{NUMERICAL EXPERIMENTS}

In this section, we demonstrate the methods of Section $\mathrm{V}$ with some numerical experiments. In Sections VI-A to VI-C, we work with tractable models where the partition function can be computed exactly via the junction tree algorithm [49]; larger grids are considered in Section VI-D

\section{A. Comparison with Deterministic Algorithms}

We first consider the Potts model with $q=3$ on an $8 \times 8$ grid with periodic boundary conditions. For this size of grid, we were able to compute the exact value of the partition function.

In Figs. 6 and 7, we compare the accuracy of the proposed methods with three standard deterministic algorithms: BP, GBP [9], [50], [51], and TreeEP [10]. These three algorithms turned out to perform best, in our setting, among all deterministic methods implemented in [52]. (Among the different versions of GBP in [52], we selected the one with the best performance.)

The accuracy of the proposed Monte Carlo methods depends on the number of samples, but the result is exact (with

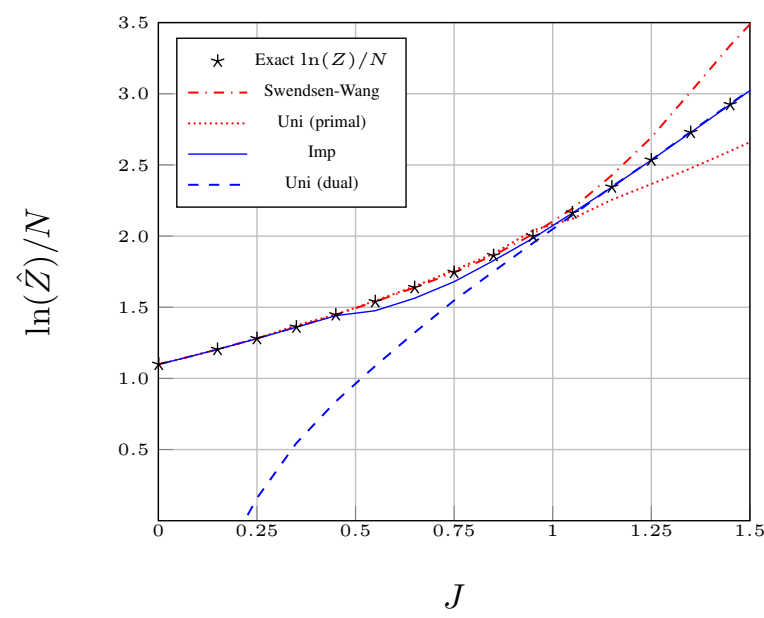

Fig. 8: Comparison with uniform sampling and the SwendsenWang algorithm: experimental results for a Potts model with $q=3, N=8 \times 8$, periodic boundary conditions, and constant couplings $J$. The plot shows $\ln (\hat{Z}) / N$ as a function of $J$.

probability one) in the limit of infinitely many samples. By contrast, the deterministic algorithms (BP, GBP, and TreeEP) yield approximations whose accuracy is not improved beyond convergence. However, it should be emphasized that deterministic algorithms converge much faster than our Monte Carlo methods.

Figs. 6 and 7 show the relative error

$$
\frac{|\log \hat{Z}-\log Z|}{\log Z}
$$

for the different estimates $\hat{Z}$. The labels "Uni" and "Imp" refer to uniform sampling as in Section $\mathrm{V}-\mathrm{A}$ and importance sampling as in Section V-B, respectively.

In Fig. 6, the couplings $J_{e}=J$ are constant. For the proposed Monte Carlo methods, $\log \hat{Z}$ in 51 is averaged over 50 trials, each with $L=10^{8}$ samples (taking about two minutes on a $2 \mathrm{GHz}$ Intel Xeon $\mathrm{CPU}$ ).

In Fig. 7, the couplings are chosen randomly according to a half-normal distribution: $J_{e}=\left|J_{e}^{\prime}\right|$ with $J_{e}^{\prime} \stackrel{\text { i.i.d. }}{\sim} \mathcal{N}\left(0, \sigma^{2}\right)$ for all $e \in \mathcal{E}$. The spanning tree was chosen according to the heuristic strategy proposed in Section V-D. The plot shows the relative error (51), where $\log Z$ is averaged over 25 independent realizations of the couplings and for every realization, $\log \hat{Z}$ is averaged over $L=10^{8}$ samples.

Both Figs. 6 and 7 clearly show that, for sufficiently strong couplings (i.e., at low temperature), the importance sampling algorithm (39) yields much better estimates of the partition function than the other algorithms. In particular, from Fig. 7 we observe that importance sampling outperforms the second best approach (TreeEP) by more than two orders of magnitude for $\sigma^{2}>1.5$. The accuracy of the proposed methods can still be improved, of course, by increasing the number of samples.

\section{B. Comparison with Standard Monte Carlo Methods}

We again consider the Potts model with $q=3$ on an $8 \times 8$ grid with periodic boundary conditions. In Fig. 8 , we compare 


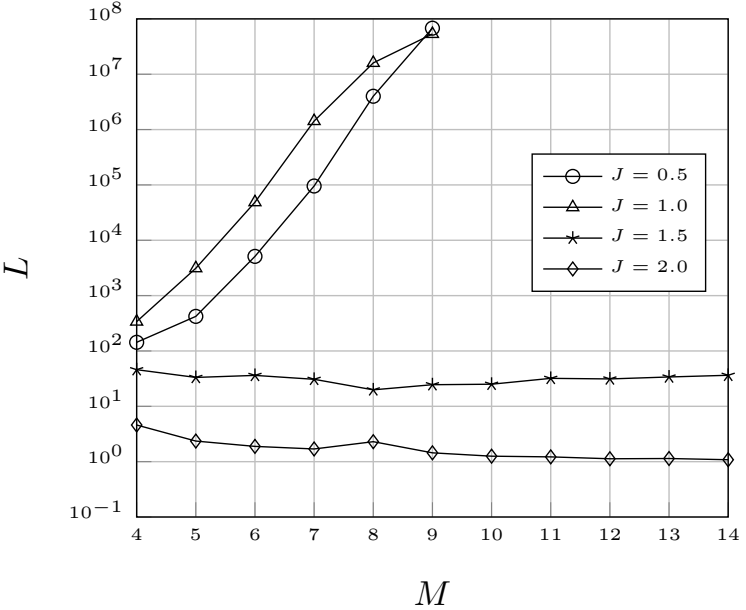

Fig. 9: Number of required samples as a function of the width of the grid $M$ to achieve a relative error 51 of $10^{-2}$, for a 2D Potts model with $q=3$, free boundary conditions, and constant couplings $J$.

the proposed methods with two Monte Carlo methods that operate in the primal Potts model. The first of these (standard) Monte Carlo algorithms (labeled "Uni (primal)" in Fig. 8) is a baseline algorithm: we use uniform samples $\mathbf{x}^{(1)}, \ldots, \mathbf{x}^{(L)} \in$ $\mathcal{X}^{N}$ and form the (unbiased) estimate

$$
\hat{Z}^{\mathrm{Uni}}=\frac{q^{N}}{L} \sum_{\ell=1}^{L} f\left(\mathbf{x}^{(\ell)}\right)
$$

with $f$ as in (7).

The second standard Monte Carlo algorithm uses the Swendsen-Wang algorithm [34] to obtain samples $\mathbf{x}^{(1)}, \ldots, \mathbf{x}^{(L)} \in \mathcal{X}^{N}$ according to the Boltzmann distribution (4). From these samples, we form the Ogata-Tanemura estimate

$$
\hat{Z}_{\mathrm{OT}}=\left(\frac{1}{L q^{N}} \sum_{\ell=1}^{L} \frac{1}{f\left(\mathbf{x}^{(\ell)}\right)}\right)^{-1},
$$

which satisfies $\mathrm{E}\left[\hat{Z}_{\mathrm{OT}}^{-1}\right]=Z^{-1}$. For more details on the OgataTanemura estimator, see [53], [32].

Fig. 8 shows the estimated $\ln (Z) / N$, where the results were obtained by averaging over 50 trials, each with $L=10^{8}$ samples. It is clear from Fig. 8 that importance sampling as in Section V-B works well for large couplings (low temperatures), where both standard Monte Carlo algorithms fail.

We do not here compare the proposed methods with annealed (i.e., multi-temperature) Monte Carlo methods [54]: since, in principle, annealing can also be used in the dual normal factor graph; the advantage of the dual graph over the primal graph at low temperatures extends also to Monte Carlo methods with annealing.

\section{Scaling Behavior of the Importance Sampling Algorithm}

We analyze the performance of the importance sampling algorithm in the dual normal factor graph in terms of the required number of samples $L$ to achieve a given relative error

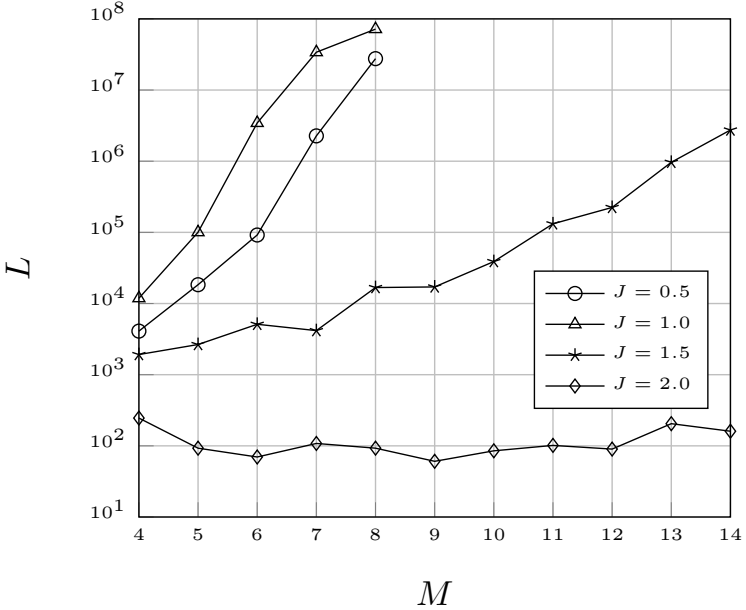

Fig. 10: Number of required samples as a function of the width of the grid $M$ to achieve a relative error 51 of $10^{-3}$, for a 2D Potts model with $q=3$, free boundary conditions, and constant couplings $J$.

as a function of the width of the gird $M$. If the desired relative error was not achieved after $L=10^{8}$ samples, we stopped the simulations.

We consider a 3-state Potts model with constant couplings $J_{e}=J$, with free boundary conditions, and on an $M \times M$ grid, where up to $M=14$ we were able to compute the exact value of the partition function.

Figs. 9 and 10 show experimental results to achieve a relative error of $10^{-2}$ and $10^{-3}$, respectively. For $J=2$ (i.e., when the temperature is low enough), the number of required samples is almost independent of $M$. For $J=1.5$, to achieve a relative error of $10^{-3}$, the number of required samples increases with $M$; but it remains almost constant to achieve a relative error of $10^{-2}$. We take these results as evidence that the importance sampling algorithm is robust at low temperature. On the other hand, for weaker couplings, $L$ grows quickly as a function of $M$.

\section{Larger Grids}

Fig. 11 shows results for a fixed realization of a Potts model with $q=3$ on a grid of size $N=40 \times 40$ and with couplings $J_{e} \stackrel{\text { i.i.d. }}{\sim} \mathcal{U}[2.5,3.0]$ for all $e \in \mathcal{E}$. The plot shows $\ln (\hat{Z}) / N$ vs. the number of samples $L$ for five independent runs of the Monte Carlo algorithms. It is obvious (and unsurprising) that importance sampling converges more quickly than uniform sampling. In this example, importance sampling yields the estimate $\ln (\hat{Z}) / N \approx 5.493$.

Fig. 12 shows results obtained from importance sampling for a fixed realization of an Ising model of size $N=50 \times$ 50 and with couplings $J_{e} \stackrel{\text { i.i.d. }}{\sim} \mathcal{U}[2.0,3.5]$ for all $e \in \mathcal{E}$. The estimated $\ln (Z) / N$ is about 6.00314 .

\section{The 2D Potts Model in an External Field}

We now extend the proposed Monte Carlo methods to Potts models with an external field, where the Hamiltonian (3) (the 


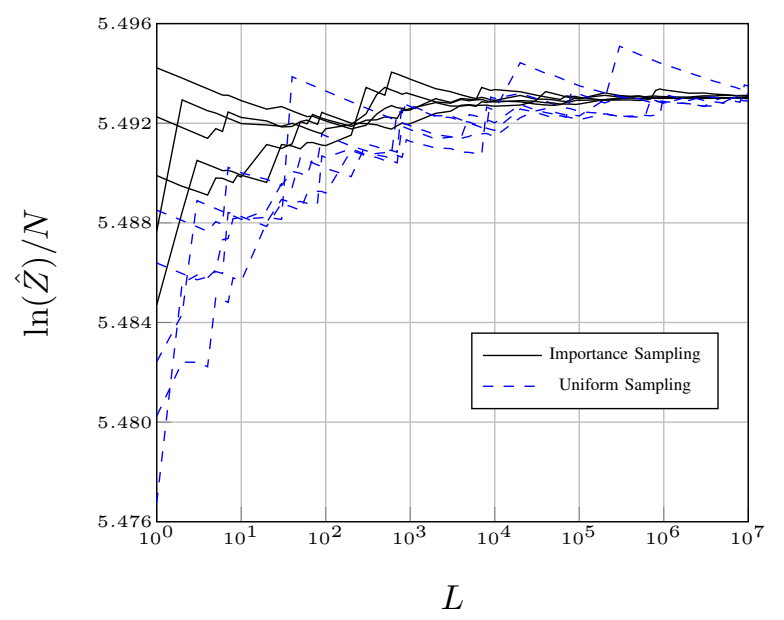

Fig. 11: Experimental results for a fixed realization of a Potts model with $q=3, N=40 \times 40$, and coupling parameters $J_{e} \stackrel{\text { i.i.d. }}{\sim} \mathcal{U}[2.5,3.0]$ for all $e \in \mathcal{E}$. The plot shows the estimated $\ln (Z) / N$ using importance sampling (solid black lines) and using uniform sampling (dashed blue lines) in the dual normal factor graph.

energy of a configuration $\mathbf{x}$ ) is generalized to

$$
\mathcal{H}(\mathbf{x})=-\sum_{(k, \ell) \in \mathcal{E}} J_{k, \ell} \cdot \delta\left(x_{k}-x_{\ell}\right)-\sum_{k=1}^{N} H_{k} \cdot \delta\left(x_{k}\right),
$$

where the real parameters $H_{k}$ represent the external field. We restrict ourselves to the standard case where the external field affects the variable $x_{k}$ only if $x_{k}=0$, cf. [55. Chapt. 1].

We will also assume

$$
H_{k} \geq 0
$$

for all $k$. The partition function is

$$
Z=\sum_{\mathbf{x} \in \mathcal{X}^{N}} e^{-\mathcal{H}(\mathbf{x})}
$$

Following our approach in Section III] we can construct the the primal normal factor graph of the model as shown in Fig. 13, where the empty boxes represent (13) and the small empty boxes represent factors given by

$$
\tau_{k}\left(x_{k}\right)= \begin{cases}e^{H_{k}}, & \text { if } x_{k}=0 \\ 1, & \text { otherwise. }\end{cases}
$$

\section{A. Dual Normal Factor Graph}

The corresponding dual normal factor graph is shown in Fig. 14 The only change with respect to Fig. 4 is the additional factors $\lambda_{k}\left(\tilde{z}_{k}\right), k=1, \ldots, N$, which are the 1D Fourier transforms of the factors 57 given by

$$
\lambda_{k}\left(\tilde{z}_{k}\right)=\frac{1}{q} \sum_{x_{k}=0}^{q-1} \tau_{k}\left(x_{k}\right) e^{-\mathrm{i} 2 \pi x_{k} \tilde{z}_{k} / q}
$$

\footnotetext{
${ }^{2}$ Here, in contrast to 17 , a local scale factor $1 / q$ is included in the definition of the 1D Fourier transform. For the 2D torus, this makes the scale factor $Z_{\mathrm{d}} / Z$ equal to $q^{N}$ in Potts models with or without an external field. If we do not include the local scale factor $1 / q$ in 58 , we need to distinguish between two cases: $Z_{\mathrm{d}}=q^{N} Z$ for the 2D torus without an external field, and $Z_{\mathrm{d}}=q^{2 N} Z$ for the $2 \mathrm{D}$ torus in the presence of an external field.
}

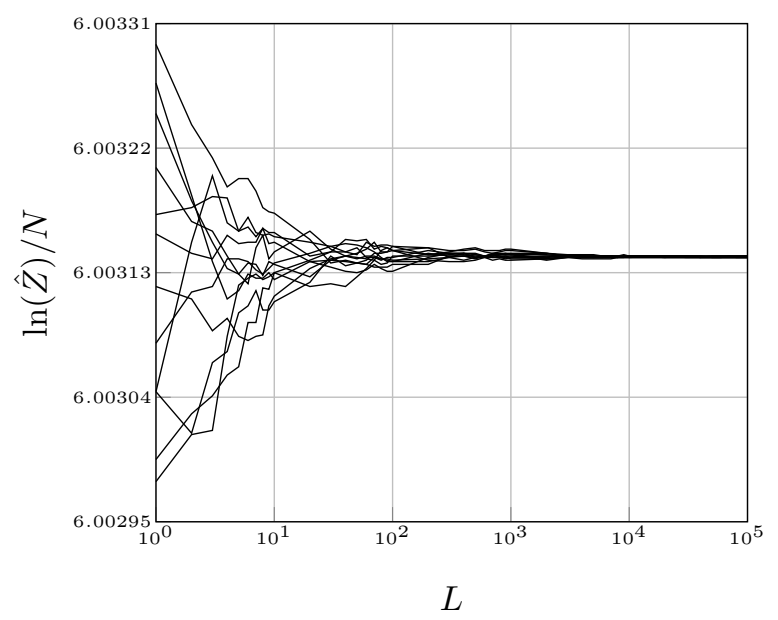

Fig. 12: Experimental results for a fixed realization of an Ising model with $N=50 \times 50$ and couplings $J_{e} \stackrel{\text { i.i.d. }}{\sim} \mathcal{U}[2.0,3.5]$ for all $e \in \mathcal{E}$. The plot shows the estimated $\ln (Z) / N$ using importance sampling in the dual normal factor graph.

Thus

$$
\lambda_{k}\left(\tilde{z}_{k}\right)= \begin{cases}\frac{e^{H_{k}}-1+q}{q}, & \text { if } \tilde{z}_{k}=0 \\ \frac{e^{H_{k}}-1}{q}, & \text { otherwise. }\end{cases}
$$

Note that 59 is nonnegative due to (55).

Again, from the scale factor 21, for the $2 \mathrm{D}$ torus we have

$$
Z_{\mathrm{d}}=q^{N} Z \text {. }
$$

\section{B. Partitioning the Variables}

For the Potts model in an external field, the variables in the dual normal factor graph consist of $\tilde{\mathbf{Y}}=\left\{\tilde{Y}_{e}: e \in \mathcal{E}\right\}$ and $\tilde{\mathbf{Z}}=\left\{\tilde{Z}_{k}: k \in\{1, \ldots, N\}\right\}$. Again, we partition these variables into $(\tilde{\mathbf{Y}}, \tilde{\mathbf{Z}})_{\mathcal{T}}$ and $(\tilde{\mathbf{Y}}, \tilde{\mathbf{Z}})_{\mathcal{\mathcal { T }}}$ such that, in any valid configuration, the variables in $(\tilde{\mathbf{Y}}, \tilde{\mathbf{Z}})_{\overline{\mathcal{T}}}$ are linearly independent and the variables in $(\tilde{\mathbf{Y}}, \tilde{\mathbf{Z}})_{\mathcal{T}}$ are fully determined by $(\tilde{\mathbf{Y}}, \tilde{\mathbf{Z}})_{\overline{\mathcal{T}}}$ via a linear transformation. However, the variables $\tilde{\mathbf{Z}}$ are not independent.

Proposition 2. In every valid configuration, it holds that

$$
\sum_{k=1}^{N} \tilde{z}_{k}=0
$$

Proof: Because of the zero-sum constraints on the vertices of the dual normal factor graph, each $\tilde{z}_{k}$ (in any valid configuration) can be written as the sum of the variables attached to the corresponding zero-sum indicator function. However, each variable will appear exactly twice in 61, once as $y_{e}$ and once as $-y_{e}$. This completes the proof.

In the absence of an external field, the number of valid configurations in the dual normal factor graph of the Potts model is $q^{|\overline{\mathcal{T}}|}$, cf. Section IV-A. Furthermore, according to Proposition 2, adding an external field increases the number of independent variables (free components) by $N-1$, and 


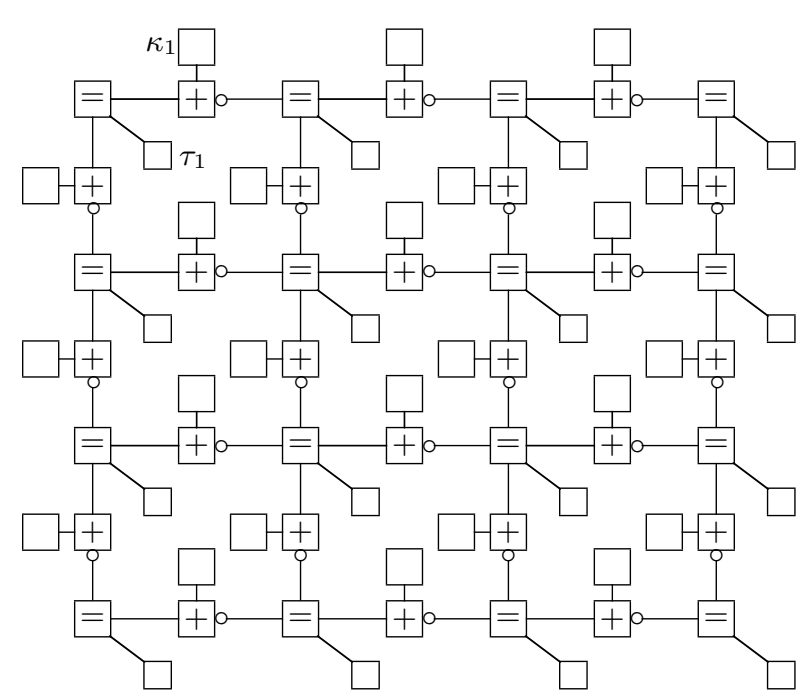

Fig. 13: Primal normal factor graph of the 2D Potts model in an external field. The only change with respect to Fig. 3 is the additional factors $\tau_{k}$ given by (57). The periodic boundary conditions are not shown.

thus increases the number of valid configurations to $q^{|\overline{\mathcal{T}}|+N-1}$. Therefore, for the 2D torus in an external field, the number of valid configurations in the dual normal factor graph is $q^{2 N}$, whereas it is $q^{N}$ in the primal normal factor graph.

Both the uniform sampling algorithm and the importance sampling algorithm of Section $\mathrm{V}$ can be adapted to the present setting. We describe only the latter for two such partitionings. The first partitioning is suitable for models in the low-temperature regime, and the second one is designed for models that are in the presence of a strong external field. The performance of the proposed algorithms depends on the choice of the partitioning, as will be illustrated by our numerical experiments in Section VII-E

\section{Importance Sampling for Models at Low Temperature}

An obvious choice for $(\tilde{\mathbf{Y}}, \tilde{\mathbf{Z}})_{\overline{\mathcal{T}}}$ with the required properties includes $\tilde{\mathbf{Y}}_{\mathcal{T}}$ (i.e., a spanning tree as in Section IV-A and $N-1$ components of $\tilde{\mathbf{Z}}$, i.e.,

$$
(\tilde{\mathbf{Y}}, \tilde{\mathbf{Z}})_{\overline{\mathcal{T}}}=\left(\tilde{\mathbf{Y}}_{\overline{\mathcal{T}}}, \tilde{\mathbf{Z}} \backslash \tilde{Z}_{1}\right),
$$

which implies

$$
(\tilde{\mathbf{Y}}, \tilde{\mathbf{Z}})_{\mathcal{T}}=\left(\tilde{\mathbf{Y}}_{\mathcal{T}}, \tilde{Z}_{1}\right)
$$

The quantities $\Gamma_{\overline{\mathcal{T}}}$ and $\Gamma_{\mathcal{T}}$ from 25 and 26 are then generalized to

$$
\Gamma_{\overline{\mathcal{T}}}\left((\tilde{\mathbf{y}}, \tilde{\mathbf{z}})_{\overline{\mathcal{T}}}\right)=\prod_{e \in \overline{\mathcal{T}}} \gamma_{e}\left(\tilde{y}_{e}\right) \prod_{k=2}^{N} \lambda_{k}\left(\tilde{z}_{k}\right)
$$

and

$$
\Gamma_{\mathcal{T}}\left((\tilde{\mathbf{y}}, \tilde{\mathbf{z}})_{\mathcal{T}}\right)=\lambda_{1}\left(\tilde{z}_{1}\right) \prod_{e \in \mathcal{T}} \gamma_{e}\left(\tilde{y}_{e}\right),
$$

respectively.

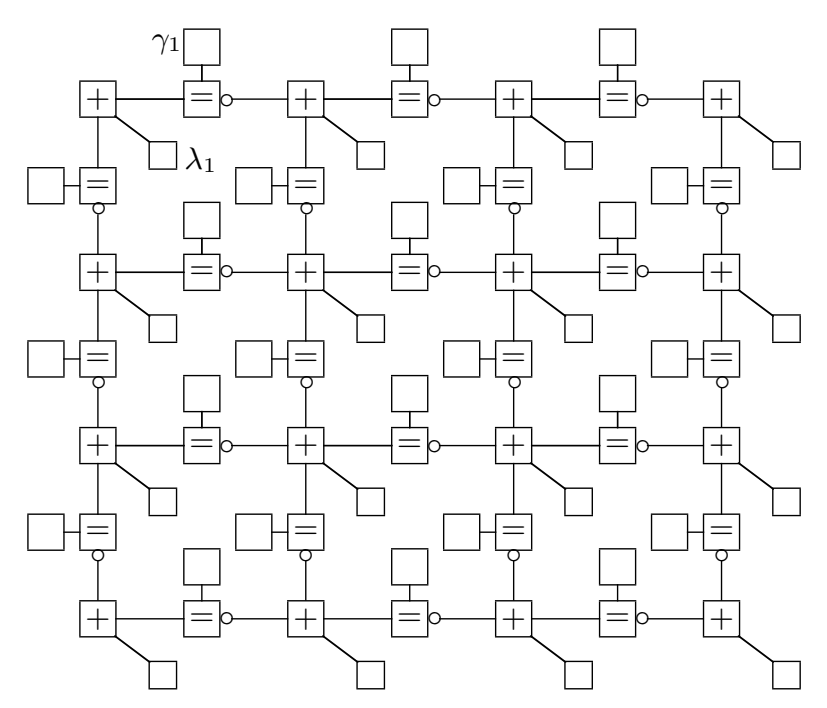

Fig. 14: Dual normal factor graph of the 2D Potts model in an external field. The only change with respect to Fig. 4 is the additional factors $\lambda_{k}$ given by (59). The periodic boundary conditions are not shown.

The quantity $Z_{\overline{\mathcal{T}}}$ from 37 is generalized to

$$
\begin{aligned}
Z_{\overline{\mathcal{T}}} & =\sum_{(\tilde{\mathbf{y}}, \tilde{\mathbf{z}})_{\overline{\mathcal{T}}}} \Gamma_{\overline{\mathcal{T}}}\left((\tilde{\mathbf{y}}, \tilde{\mathbf{z}})_{\overline{\mathcal{T}}}\right) \\
& =\left(\prod_{e \in \overline{\mathcal{T}}} \sum_{\xi=0}^{q-1} \gamma_{e}(\xi)\right)\left(\prod_{k=2}^{N} \sum_{\xi^{\prime}=0}^{q-1} \lambda_{k}\left(\xi^{\prime}\right)\right) \\
& =\left(\prod_{e \in \overline{\mathcal{T}}} q e^{J_{e}}\right)\left(\prod_{k=2}^{N} e^{H_{k}}\right) \\
& =q^{|\overline{\mathcal{T}}|} \exp \left(\sum_{e \in \overline{\mathcal{T}}} J_{e}+\sum_{k=2}^{N} H_{k}\right) .
\end{aligned}
$$

The algorithm then goes as follows.

1) Generate $L$ independent samples $(\tilde{\mathbf{x}}, \tilde{\mathbf{y}})_{\overline{\mathcal{T}}}^{(1)}, \ldots,(\tilde{\mathbf{y}}, \tilde{\mathbf{z}})_{\overline{\mathcal{T}}}^{(L)}$ from the distribution

$$
p_{\overline{\mathcal{T}}}\left((\tilde{\mathbf{y}}, \tilde{\mathbf{z}})_{\overline{\mathcal{T}}}\right)=\frac{\Gamma_{\overline{\mathcal{T}}}\left((\tilde{\mathbf{y}}, \tilde{\mathbf{z}})_{\overline{\mathcal{T}}}\right)}{Z_{\overline{\mathcal{T}}}}
$$

2) For each sample $(\tilde{\mathbf{y}}, \tilde{\mathbf{z}})_{\overline{\mathcal{T}}}^{(\ell)}$, compute its unique extension $(\tilde{\mathbf{y}}, \tilde{\mathbf{z}})_{\mathcal{T}}^{(\ell)}$ to a valid configuration $(\tilde{\mathbf{y}}, \tilde{\mathbf{z}})^{(\ell)}$, including $\tilde{z}_{1}$, which can be computed as

$$
\tilde{z}_{1}=-\sum_{m=2}^{N} \tilde{z}_{m}
$$

from 61).

3) Compute the estimate

$$
\hat{Z}_{\mathrm{d}}^{\mathrm{Imp}}=\frac{Z_{\overline{\mathcal{T}}}}{L} \sum_{\ell=1}^{L} \Gamma_{\mathcal{T}}\left((\tilde{\mathbf{y}}, \tilde{\mathbf{z}})_{\mathcal{T}}^{(\ell)}\right) .
$$

The estimate (72) is easily verified to be unbiased, cf. (43). Creating the samples $(\tilde{\mathbf{y}}, \tilde{\mathbf{z}})_{\overline{\mathcal{T}}}^{(\ell)}$ in Step 1 is straightforward 

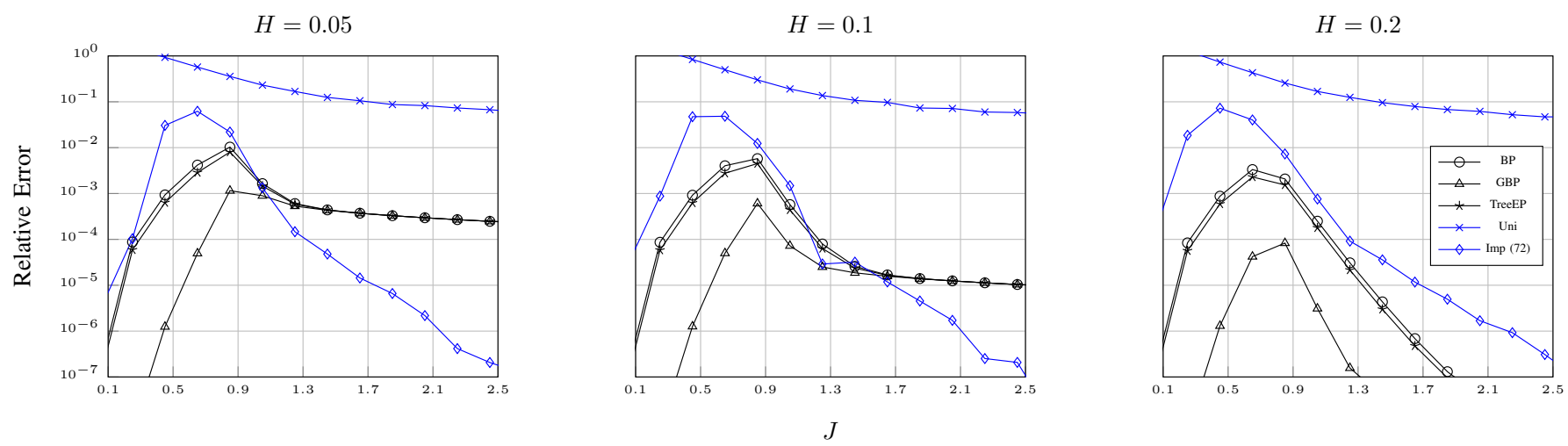

Fig. 15: Comparison with deterministic algorithms (BP, GBP, and TreeEP): experimental results for a Potts model with $q=3$, $N=8 \times 8$, constant coupling parameter $J$, and in a constant external field $H$. The plots show the relative error $(51)$ as a function of $J$. Left: $H=0.05$; middle: $H=0.1$; right: $H=0.2$.

since the distribution in 70 decomposes into a product: first sample the components $\tilde{y}_{e}^{(\ell)}, e \in \overline{\mathcal{T}}$, of each sample independently according to 38 , then sample the components $\tilde{z}_{2}^{(\ell)}, \ldots, \tilde{z}_{N}^{(\ell)}$ of each sample independently according to

$$
\begin{aligned}
& P\left(\tilde{z}_{k}^{(\ell)}=\xi\right) \\
& \quad= \begin{cases}\frac{1+(q-1) e^{-H_{k}}}{q}, & \text { if } \xi=0 \\
\frac{1-e^{-H_{k}}}{q}, & \text { for } \xi=1,2, \ldots, q-1 .\end{cases}
\end{aligned}
$$

With this choice of partitioning, it can be verified that 48 vanishes when $e^{J_{e}} \gg q$ for $e \in \mathcal{T}$.

One can design a slightly different algorithm with rejections by drawing all the components $\tilde{z}_{1}^{(\ell)}, \tilde{z}_{2}^{(\ell)}, \ldots, \tilde{z}_{N}^{(\ell)}$ according to (73); but accept only the samples that satisfy (61). The corresponding partitioning in the dual normal factor graph is

$$
(\tilde{\mathbf{Y}}, \tilde{\mathbf{Z}})_{\overline{\mathcal{T}}}=\left(\tilde{\mathbf{Y}}_{\overline{\mathcal{T}}}, \tilde{\mathbf{Z}}\right)
$$

Accordingly

$$
\Gamma_{\overline{\mathcal{T}}}\left((\tilde{\mathbf{y}}, \tilde{\mathbf{z}})_{\overline{\mathcal{T}}}\right)=\prod_{e \in \overline{\mathcal{T}}} \gamma_{e}\left(\tilde{y}_{e}\right) \prod_{k=1}^{N} \lambda_{k}\left(\tilde{z}_{k}\right)
$$

and

$$
\Gamma_{\mathcal{T}}\left((\tilde{\mathbf{y}}, \tilde{\mathbf{z}})_{\mathcal{T}}\right)=\prod_{e \in \mathcal{T}} \gamma_{e}\left(\tilde{y}_{e}\right)
$$

For more details, see [3].

\section{Importance Sampling for Models in a Strong External Field}

We assume another partitioning of $(\tilde{\mathbf{Y}}, \tilde{\mathbf{Z}})$ given by

$$
(\tilde{\mathbf{Y}}, \tilde{\mathbf{Z}})_{\overline{\mathcal{T}}}=\tilde{\mathbf{Y}}
$$

which implies

$$
(\tilde{\mathbf{Y}}, \tilde{\mathbf{Z}})_{\mathcal{T}}=\tilde{\mathbf{Z}}
$$

This partitioning generalizes $\Gamma_{\overline{\mathcal{T}}}$ and $\Gamma_{\mathcal{T}}$ to

$$
\Gamma_{\overline{\mathcal{T}}}(\tilde{\mathbf{y}})=\prod_{e \in \mathcal{E}} \gamma_{e}\left(\tilde{y}_{e}\right)
$$

and

$$
\Gamma_{\mathcal{T}}(\tilde{\mathbf{z}})=\prod_{k=1}^{N} \lambda_{k}\left(\tilde{z}_{k}\right) .
$$

The partition function $Z_{\overline{\mathcal{T}}}$ is then generalized to

$$
\begin{aligned}
Z_{\overline{\mathcal{T}}} & =\sum_{\tilde{\mathbf{y}}} \Gamma_{\overline{\mathcal{T}}}(\tilde{\mathbf{y}}) \\
& =q^{|\mathcal{E}|} \exp \left(\sum_{e \in \mathcal{E}} J_{e}\right) .
\end{aligned}
$$

The importance sampling algorithm goes as follows.

1) Generate $L$ independent samples $\tilde{\mathbf{y}}^{(1)}, \ldots, \tilde{\mathbf{y}}^{(L)}$ according to

$$
p_{\overline{\mathcal{T}}}(\tilde{\mathbf{y}})=\frac{\Gamma_{\overline{\mathcal{T}}}(\tilde{\mathbf{y}})}{Z_{\overline{\mathcal{T}}}} .
$$

2) From each sample $\tilde{\mathbf{y}}^{(\ell)}$, compute $\tilde{\mathbf{z}}^{(\ell)}$.

3) Compute the unbiased estimate

$$
\hat{Z}_{\mathrm{d}}^{\mathrm{Imp}}=\frac{Z_{\overline{\mathcal{T}}}}{L} \sum_{\ell=1}^{L} \Gamma_{\mathcal{T}}\left(\tilde{\mathbf{z}}^{(\ell)}\right) .
$$

In this setting, it can be verified that (48) vanishes in the limit of the strong field (i.e., for $e^{H} \gg q$ ). For more details, see [2]. The partitioning in (77) and 78) will also be used in Section VIII to establish connections between the dual normal factor graph representation of the 2D Ising model in an external field and the high-temperature series expansions of the partition function.

\section{E. Numerical Experiments}

Experimental results for a Potts model with $N=8 \times 8$, $q=3$, and periodic boundary conditions are shown in Figs. 15 and 16. for constant coupling parameter $J_{e}=J$ and for constant external field $H_{k}=H$. The partition function is computed exactly via the junction tree algorithm, which allows to compare the accuracy of different algorithms. For the Monte Carlo algorithms, the estimates are averaged over 50 trials, each with $L=10^{8}$ samples. The accuracy of the estimates is then compared with deterministic algorithms: BP, GBP, and 
TreeEP as in Section VI-A The figures show the relative error (51) as a function of $J$.

Fig. 15] shows results for $H=0.05$ (left), $H=0.1$ (middle), and $H=0.2$ (right). We find that GBP works well, especially for $H=0.2$; but the importance sampling algorithm 72 is more accurate at low temperatures (i.e., for large $J$ ) and in weak external fields.

For $H=1.0$, experimental results are shown in Fig. 16 , where we consider both importance sampling algorithms proposed in (72) and (84). As expected, we observe that the importance sampler in (84) outperforms (72) for stronger external fields (stronger compared to the coupling parameter). However, GBP performs extremely well in this setting, indeed its relative error is below $10^{-7}$ in the whole range.

The accuracy of the importance sampling algorithms can be improved, of course, by increasing the number of samples.

\section{The 2D IsIng Model in AN ExTERnAl Field, THE HIGH-TEMPERATURE SERIES EXPANSION, AND THE SUBGRAPHS-WORLD PROCESS}

In this section, we show the equivalence between the valid configurations in the dual normal factor graph and the configurations in Jerrum and Sinclair subgraphs-world process [24] for a ferromagnetic Ising model on a 2D torus. Following [24], we restrict our focus to Ising models in a constant external field $H$.

For this model, the energy of a configuration $\mathrm{x} \in \mathcal{X}^{N}$ is given by the Hamiltonian [8, Chapter 3]

$$
\begin{array}{r}
\mathcal{H}(\mathbf{x})=-\sum_{(k, \ell) \in \mathcal{E}} J_{k, \ell} \cdot\left(2 \delta\left(x_{k}-x_{\ell}\right)-1\right) \\
-H \sum_{k=1}^{N}\left(1-2 \delta\left(x_{k}\right)\right) .
\end{array}
$$

The primal normal factor graph of the model is shown in Fig. 13, where the empty boxes represent (16) and the small empty boxes represent the factors

$$
\tau\left(x_{k}\right)= \begin{cases}e^{-H}, & \text { if } \tilde{x}_{k}=0 \\ e^{H}, & \text { if } \tilde{x}_{k}=1 .\end{cases}
$$

Fig. 14 shows the dual normal factor graph of the 2D Ising model in an external field, where the empty boxes represent (19) and the small empty boxes represent the factors $\lambda\left(\tilde{z}_{k}\right)$, which are the 1D Fourier transforms of 86 , and are given by

$$
\lambda\left(\tilde{z}_{k}\right)= \begin{cases}\cosh (H), & \text { if } \tilde{z}_{k}=0 \\ -\sinh (H), & \text { if } \tilde{z}_{k}=1 .\end{cases}
$$

Again, for the Ising model, the "o" symbols are immaterial and can be removed from normal factor graphs.

Since the partition function is invariant under the change of sign of the external field [7. Chapter 1], we will assume $H \leq 0$. Therefore, factors 87 are nonnegative. The invariance of the partition function under the change of sign of $H$ is implied by Proposition 2, as in any valid configuration in the dual normal factor graph, it holds that

$$
\sum_{k=1}^{N} \tilde{z}_{k}=0
$$

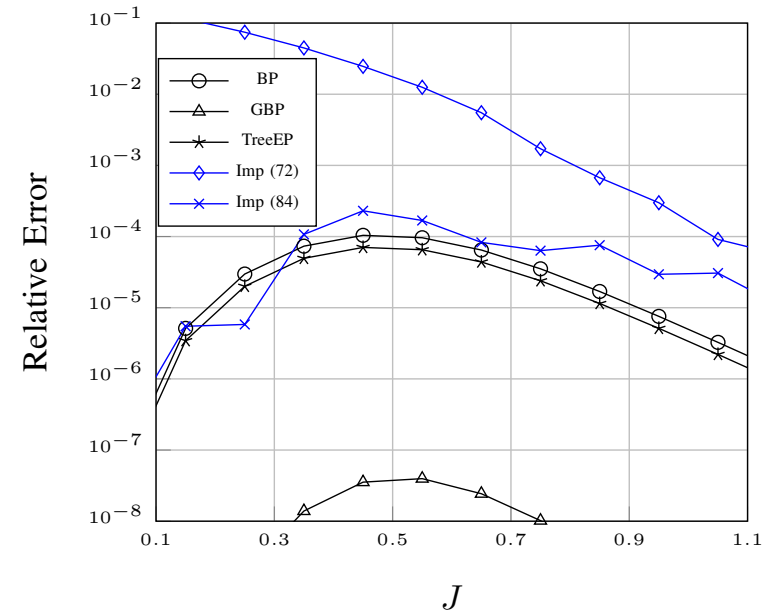

Fig. 16: Comparison with deterministic algorithms (BP, GBP, and TreeEP): experimental results for a Potts model with $q=3, N=8 \times 8$, constant coupling parameter $J$, and in a constant external field $H=1.0$. The plots show the relative error (51) as a function of $J$.

i.e., the Hamming weight of $\tilde{\mathbf{Z}}$ is always even, where the Hamming weight of a configuration is the number of nonzero components of that configuration [56]. Indeed, $\prod_{k=1}^{N} \lambda\left(\tilde{z}_{k}\right)$ takes on the same positive value regardless of the sign of $H$.

In [24], the authors propose a Markov chain (called the subgraphs-world process), which is defined on the set of edges $\mathcal{W} \subseteq \mathcal{E}$ of the interaction graph of the model (as in Fig. 14). The scheme of Jerrum and Sinclair then uses the following expansion of the partition function in powers of $\tanh (H)$ and $\tanh (J)$

$$
Z=A \sum_{\mathcal{W} \subseteq \mathcal{E}} \tanh (H)^{|\operatorname{odd}(\mathcal{W})|} \prod_{(k, \ell) \in \mathcal{W}} \tanh \left(J_{e}\right),
$$

where odd $(\mathcal{W})$ denotes the set of all odd-degree vertices in the subgraph of $\mathcal{E}$ induced by $\mathcal{W}$, and

$$
A=(2 \cosh (H))^{N} \prod_{(k, \ell) \in \mathcal{W}} \cosh \left(J_{e}\right) .
$$

The sum in 89 is known as the high-temperature series expansion in statistical physics [57], [8, p. 94].

In the dual normal factor graph, we adopt the partitioning of $(\tilde{\mathbf{Y}}, \tilde{\mathbf{Z}})$ proposed in 777 and 78 . We can thus freely choose the variables $\tilde{\mathbf{Y}}=\left\{\bar{Y}_{e}: e \in \mathcal{E}\right\}$, and therefrom compute the variables $\tilde{\mathbf{Z}}=\left\{\tilde{Z}_{k}: k \in\{1, \ldots, N\}\right\}$.

The partition function $Z_{\mathrm{d}}$ can then be written as

$$
\begin{aligned}
Z_{\mathrm{d}}= & \sum_{\operatorname{valid}(\tilde{\mathbf{y}}, \tilde{\mathbf{z}})} \prod_{k=1}^{N} \lambda\left(\tilde{z}_{k}\right) \prod_{e \in \mathcal{E}} \gamma_{e}\left(\tilde{y}_{e}\right) \\
= & 2^{|\mathcal{E}|} \cosh (H)^{N} \prod_{e \in \mathcal{E}} \cosh \left(J_{e}\right) . \\
& \sum_{\operatorname{valid}(\tilde{\mathbf{y}}, \tilde{\mathbf{z}})} \prod_{k=1}^{N} \tanh (|H|)^{\tilde{z}_{k}} \prod_{e \in \mathcal{E}} \tanh \left(J_{e}\right)^{\tilde{y}_{e}} .
\end{aligned}
$$


In a $2 \mathrm{D}$ torus $|\mathcal{E}|=2 N$, thus

$$
Z_{\mathrm{d}}=2^{N} A \sum_{\operatorname{valid}(\tilde{\mathbf{y}}, \tilde{\mathbf{z}})} \tanh (|H|)^{\sum_{k=1}^{N} \tilde{z}_{k}} \prod_{e \in \mathcal{E}} \tanh \left(J_{e}\right)^{\tilde{y}_{e}},
$$

where $A$ is as in 90.

Accordingly, we define $\mathcal{S} \subseteq \mathcal{E}$ as

$$
\mathcal{S}(\tilde{\mathbf{y}}) \triangleq\left\{e: \tilde{Y}_{e}=1\right\} .
$$

Here, as $\tilde{\mathbf{y}}$ runs over the configurations in the dual normal factor graph, $\mathcal{S}(\tilde{\mathbf{y}})$ runs over all the $2^{|\mathcal{E}|}$ subsets of $\mathcal{E}$. Let us consider the subgraph of $\mathcal{E}$ induced by $\mathcal{S}$, in which $\tilde{z}_{k}=1$ if it is connected to a zero-sum indicator function with odd degree, and $\tilde{z}_{k}=0$ otherwise. Therefore, $\sum_{k=1}^{N} \tilde{z}_{k}$ counts the number of odd-degree vertices in $\mathcal{S}$. Thus, from 93 we obtain

$$
Z_{\mathrm{d}}=2^{N} A \sum_{\mathcal{S} \subseteq \mathcal{E}} \tanh (|H|)^{|\operatorname{odd}(\mathcal{S})|} \prod_{e \in \mathcal{S}(\tilde{\mathbf{y}})} \tanh \left(J_{e}\right) .
$$

After applying the scale factor (60) in (95), we will obtain the high-temperature series expansion of the partition function given by 89 . We conclude that the configurations in the subgraphs-world process coincide with the valid configurations in the dual normal factor graph of the Ising model in an external field. (The number of vertices with odd degree in the subgraph $\mathcal{S}$ is always even, which is also implied by Proposition 2, )

The scheme of Jerrum and Sinclair works on a Markov chain whose states are configurations of the subgraphs-world and whose stationary distribution is given by $p_{\mathrm{d}}$, cf. Section V Transitions occur between states that differ in a single edge according to the Metropolis rule. Remarkably, the mixing time of the proposed Markov chain is only polynomial in the size of the model at all temperatures. Indeed, a rigorous analysis shows that the expected running time of the generator for the subgraphs-world configurations is upper bounded by $O\left(|E|^{2} N^{8}\left(\log \epsilon^{-1}+|E|\right)\right)$, where $\epsilon$ is the confidence parameter. The scheme is randomized, i.e., it provides approximations to the partition function, which fall within arbitrary small error bounds with high probability [24, Section 4].

Our proposed unbiased Monte Carlo methods in the dual normal factor graph draw independent samples according to an auxiliary distribution. The partition function is then estimated by averaging according to the importance sampling weights of the independent samples, cf. (39). Monte Carlo methods of this paper work particularly well in the low-temperature regime.

The main focus of this paper is on the (nonbinary) Potts model, where Monte Carlo methods in the dual normal factor graph outperform the state-of-the-art methods at low temperatures (with no external field or in a weak external field). However, approximating the partition function of the ferromagnetic Potts model is already as hard as approximating the number of independent sets in a bipartite graph, which is among the presumably intractable (the \#BIS-hardness) problems. For more details see [25]-[27].

\section{CONClusion}

We reviewed representations of the Ising and Potts models of statistical physics in terms of normal factor graphs, and further explored the idea that Monte Carlo algorithms in the dual normal factor graph can yield good estimates of the partition function at low temperatures. Specifically, we proposed and investigated such algorithms for the ferromagnetic Potts model, and we observed good convergence for strong couplings (i.e., at low temperatures). In our numerical experiments, for the 2D ferromagnetic Potts models, the importance sampling algorithms in the dual normal factor graph yield more accurate estimates at low temperatures (and with no, or weak, external field) than the state-of-the-art deterministic and Monte Carlo methods. We expect such Monte Carlo methods in the dual normal factor graph to work well also for threedimensional grids and in graphical models with more general topologies. Using deterministic algorithms (e.g., GBP and TreeEP) in the dual graph is certainly possible, but it has not been tried yet.

We also showed the equivalence between the valid configurations in the dual normal factor graph and the terms that appear in the high-temperature series expansion of the partition function of the ferromagnetic Ising model in an external field, and discussed connections with the subgraphs-world process in the randomized approximate scheme of Jerrum and Sincalir.

Finally, it should be mentioned that the factors in the dual normal factor graph can, in general, be negative or even complex-valued, which could be a serious challenge for Monte Carlo methods. Such issues were avoided in this paper by considering only ferromagnetic models, but they must be faced in any attempt to deal with antiferromagnetic models, spin glasses, and computational problems in quantum information processing [58]-[61].

\section{APPENDIX A \\ Comparing THE VARIANCE OF Monte CARLo Methods \\ IN THE PRIMAL AND IN THE DUAL NORMAL FACTOR GRAPHS OF THE 2D ISING MODEL}

We compare the variance of the uniform sampling and importance sampling estimators in the primal and in the dual domains for estimating the partition function of the Ising model on a $2 \mathrm{D}$ torus, with constant coupling parameter $J$, without an external field, and in the thermodynamic limit (i.e., as $N \rightarrow \infty)$. The choice of the model and the parameters is due to the fact that the partition function is analytically available from Onsager's solution in this case, see [44], [7. Chapter 7].

For this model, the critical coupling (i.e., the phase transition) is located at

$$
J_{\mathrm{c}}=\frac{1}{2} \ln (1+\sqrt{2}) \approx 0.4407
$$

and, at criticality, the derivative of $\ln Z$ with respect to $J$ (i.e., the internal energy of the model) is given by

$$
\lim _{N \rightarrow \infty} \frac{1}{N} \frac{\partial \ln Z\left(J_{\mathrm{c}}\right)}{\partial J_{\mathrm{c}}}=\sqrt{2},
$$

see [62].

In the primal domain, the analytical solution of the partition function allows us to calculate the exact value of the variance of the uniform sampling estimator as a function of $J$. In 
the dual domain, we provide upper and lower bounds on the variance of the estimators. The derived bounds are not necessarily tight for all values of $J$, however, they are good enough to illustrate the opposite behavior of the estimators in the primal and in the dual domains.

We recall from $(23)$ and $(24)$ that for the $2 \mathrm{D}$ torus $|\mathcal{T}|=$ $N-1$ and $|\overline{\mathcal{T}}|=N+1$.

\section{A. Uniform Sampling}

Following our analysis in Section $\mathrm{V}-\mathrm{C}$, the variance of the uniform sampling estimator in the primal domain 52 can be expressed as

$$
\operatorname{Var}\left[\hat{Z}^{\mathrm{Uni}}\right] \frac{L}{Z^{2}}=\chi^{2}\left(p_{\mathrm{B}}, p_{\mathrm{u}}\right),
$$

where $p_{\mathrm{B}}(\mathbf{x})$ is the Boltzmann distribution given by (4) and $p_{\mathbf{u}}(\mathbf{x})$ is the uniform distribution over all the configurations.

It follows that

$$
\begin{aligned}
1+\operatorname{Var}\left[\hat{Z}^{\text {Uni }}\right] \frac{L}{Z(J)^{2}} & =\sum_{\mathbf{x}} \frac{p_{\mathrm{B}}(\mathbf{x})^{2}}{p_{\mathbf{u}}(\mathbf{x})} \\
& =\frac{2^{N}}{Z(J)^{2}} \sum_{\mathbf{x}} f(\mathbf{x})^{2} \\
& =2^{N} \frac{Z(2 J)}{Z(J)^{2}},
\end{aligned}
$$

where $Z(J)$ denotes the partition function evaluated at $J$, and the last step is due to the following identity

$$
Z(2 J)=\sum_{\mathbf{x}} f(\mathbf{x})^{2} .
$$

Thus, in the thermodynamic limit we obtain

$$
\begin{aligned}
\lim _{N \rightarrow \infty} & \frac{1}{N} \ln \left(1+\operatorname{Var}\left[\hat{Z}^{\mathrm{Uni}}\right] \frac{L}{Z(J)^{2}}\right)= \\
& \ln (2)+\lim _{N \rightarrow \infty} \frac{\ln Z(2 J)}{N}-\lim _{N \rightarrow \infty} \frac{2 \ln Z(J)}{N} .
\end{aligned}
$$

We use the closed-form solution of the partition function to evaluate (103) numerically as a function of $J$, which is plotted by the solid black line in Fig. 17 As expected, we observe that uniform sampling in the primal domain can provide good estimates of the partition function when $J$ is small (i.e., at high temperature), while it is an inefficient estimator for larger values of $J$ (i.e., at low temperature).

From (49, we expand the variance of the uniform sampling algorithm in the dual domain (29) as

$$
\begin{aligned}
1+\operatorname{Var}\left[\hat{Z}_{\mathrm{d}}^{\text {Uni }}\right] \frac{L}{Z_{\mathrm{d}}(J)^{2}} & =\sum_{\text {valid } \tilde{\mathbf{y}}} \frac{p_{\mathrm{d}}(\tilde{\mathbf{y}})^{2}}{p_{\mathrm{u}}(\tilde{\mathbf{y}})} \\
& =\frac{2^{|\overline{\mathcal{T}}|}}{Z_{\mathrm{d}}(J)^{2}} \sum_{\text {valid } \tilde{\mathbf{y}}} \Gamma(\tilde{\mathbf{y}})^{2} \\
& =\frac{2^{N+1}}{Z_{\mathrm{d}}(J)^{2}} R,
\end{aligned}
$$

where $R \triangleq \sum_{\text {valid } \tilde{\mathbf{y}}} \Gamma(\tilde{\mathbf{y}})^{2}$.

From 21), we have $Z_{\mathrm{d}}=2^{N} Z$. Thus

$$
1+\operatorname{Var}\left[\hat{Z}_{\mathrm{d}}^{\mathrm{Uni}}\right] \frac{L}{Z_{\mathrm{d}}(J)^{2}}=\frac{2^{-N+1}}{Z(J)^{2}} R .
$$

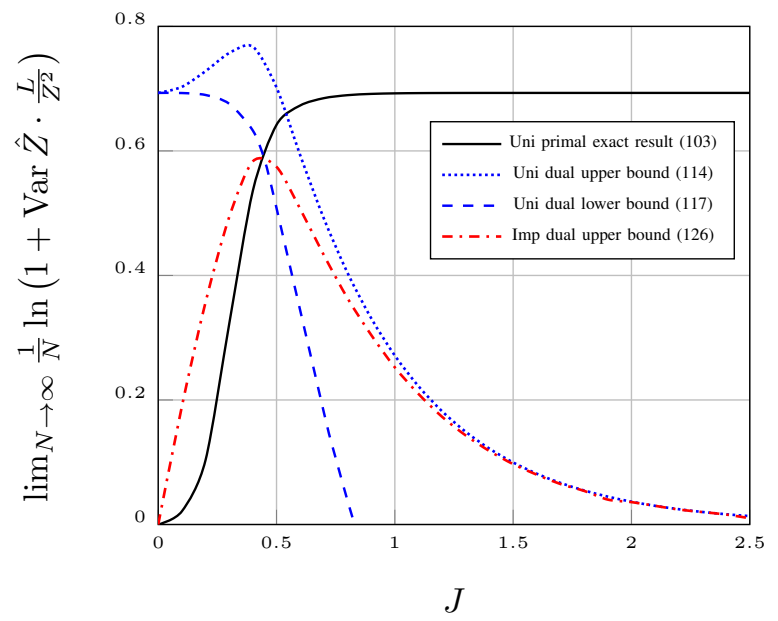

Fig. 17: Comparing the variance of Monte Carlo methods in the primal and in the dual domains for the Ising model on a 2D torus, with constant coupling $J$, and in the thermodynamic limit. The solid black line shows (103); for the uniform sampling algorithm in the dual domain the dotted blue line shows the upper bound in (114) and the dashed blue line shows the lower bound in (117); for the importance sampling algorithm in the dual domain the dashed-dotted red line shows the upper bound in 126 .

In the sequel, we will derive upper and lower bounds on $R$, which is the partition function of a dual normal factor graph (as shown in Fig. 4) with factors given by

$$
\rho\left(\tilde{y}_{e}\right)= \begin{cases}4 \cosh (J)^{2}, & \text { if } \tilde{y}_{e}=0 \\ 4 \sinh (J)^{2}, & \text { if } \tilde{y}_{e}=1 .\end{cases}
$$

Thus

$$
\begin{aligned}
R & =\sum_{\text {valid }} \prod_{\tilde{\mathbf{y}}} \prod_{e \in \mathcal{E}} \rho\left(\tilde{y}_{e}\right) \\
& \leq\left(4 \cosh (J)^{2}\right)^{|\mathcal{T}|} \sum_{\tilde{\mathbf{y}}_{\overline{\mathcal{T}}}} \prod_{e \in \overline{\mathcal{T}}} \rho\left(\tilde{y}_{e}\right) \\
& =(2 \cosh (J))^{2(N-1)} R_{\overline{\mathcal{T}}}
\end{aligned}
$$

Here, $R_{\overline{\mathcal{T}}}$ is the partition function of a subgraph of the dual normal factor graph induced by $\overline{\mathcal{T}}$, which can be computed exactly as

$$
\begin{aligned}
R_{\overline{\mathcal{T}}} & =(\rho(0)+\rho(1))^{|\overline{\mathcal{T}}|} \\
& =(4 \cosh (2 J))^{N+1} .
\end{aligned}
$$

Combining (107), (111), and (113) yields the following upper bound

$$
\begin{aligned}
\lim _{N \rightarrow \infty} & \frac{1}{N} \ln \left(1+\operatorname{Var}\left[\hat{Z}_{\mathrm{d}}^{\mathrm{Uni}}\right] \frac{L}{Z_{\mathrm{d}}(J)^{2}}\right) \leq 3 \ln (2) \\
& +\ln \left(\cosh (2 J) \cdot \cosh (J)^{2}\right)-\lim _{N \rightarrow \infty} \frac{2 \ln Z(J)}{N},
\end{aligned}
$$

which is plotted by the dotted blue line in Fig. 17 
To obtain the lower bound, we note that

$$
\begin{aligned}
R & =\sum_{\text {valid }} \prod_{\tilde{\mathbf{y}}} \rho\left(\tilde{y}_{e}\right) \\
& \geq\left(4 \cosh (J)^{2}\right)^{2 N} .
\end{aligned}
$$

Combining (107) and 116 gives the following lower bound

$$
\begin{aligned}
\lim _{N \rightarrow \infty} \frac{1}{N} \ln (1 & \left.+\operatorname{Var}\left[\hat{Z}_{\mathrm{d}}^{\mathrm{Uni}}\right] \frac{L}{Z_{\mathrm{d}}(J)^{2}}\right) \geq 3 \ln (2) \\
& +4 \ln (\cosh (J))-\lim _{N \rightarrow \infty} \frac{2 \ln Z(J)}{N},
\end{aligned}
$$

which is plotted by the dashed blue line in Fig. 17

From Fig. 17, we observe that uniform sampling in the dual domain is inefficient for small values of $J$, however, compared to uniform sampling in the primal domain, it can provide more reliable estimates of the partition function when $J$ is large. (Recall from Section $\mathrm{V}-\mathrm{C}$ that 49 vanishes as $J \rightarrow \infty$, i.e, in the low-temperature limit.)

Both estimators seem to be inefficient in the midtemperature regime and near criticality 96 .

\section{B. Importance Sampling}

From (48), the variance of the importance sampling algorithm can be expressed as

$$
\begin{aligned}
1+\operatorname{Var}\left[\hat{Z}_{\mathrm{d}}^{\operatorname{Imp}}\right] \frac{L}{Z_{\mathrm{d}}(J)^{2}} & =\sum_{\operatorname{valid} \tilde{\mathbf{y}}} \frac{p_{\mathrm{d}}(\tilde{\mathbf{y}})^{2}}{p_{\overline{\mathcal{T}}}(\tilde{\mathbf{y}})} \\
& =\frac{Z_{\overline{\mathcal{T}}}^{2}}{Z_{\mathrm{d}}(J)^{2}} \sum_{\text {valid } \tilde{\mathbf{y}}} p_{\overline{\mathcal{T}}}(\tilde{\mathbf{y}}) \Gamma_{\mathcal{T}}\left(\tilde{\mathbf{y}}_{\mathcal{T}}\right)^{2} .
\end{aligned}
$$

From 21, we have $Z_{\mathrm{d}}=2^{N} Z$. Moreover,

$$
\begin{aligned}
Z_{\overline{\mathcal{T}}} & =\sum_{\tilde{\mathbf{y}}_{\overline{\mathcal{T}}}} \Gamma_{\overline{\mathcal{T}}}\left(\tilde{\mathbf{y}}_{\overline{\mathcal{T}}}\right) \\
& =2^{|\overline{\mathcal{T}}|} e^{J|\overline{\mathcal{T}}|} \\
& =2^{N+1} e^{J(N+1)},
\end{aligned}
$$

cf. (37). From (19) and (26), we obtain

$$
\begin{aligned}
\sum_{\text {valid } \tilde{\mathbf{y}}} p_{\overline{\mathcal{T}}}(\tilde{\mathbf{y}}) \Gamma_{\mathcal{T}}\left(\tilde{\mathbf{y}}_{\mathcal{T}}\right)^{2} & \leq\left(4 \cosh (J)^{2}\right)^{|\mathcal{T}|} \\
& =(2 \cosh (J))^{2(N-1)} .
\end{aligned}
$$

Thus

$$
1+\operatorname{Var}\left[\hat{Z}_{\mathrm{d}}^{\mathrm{Imp}}\right] \frac{L}{Z_{\mathrm{d}}(J)^{2}}=\frac{2^{2 N} e^{2 J(N+1)}}{Z(J)^{2}} \cosh (J)^{2(N-1)},
$$

which, in the thermodynamic limit $N \rightarrow \infty$, gives the following upper bound

$$
\begin{aligned}
\lim _{N \rightarrow \infty} \frac{1}{N} & \ln \left(1+\operatorname{Var}\left[\hat{Z}_{\mathrm{d}}^{\operatorname{Imp}}\right] \frac{L}{Z_{\mathrm{d}}(J)^{2}}\right) \leq 2 \ln (2) \\
& +2 J+2 \ln (\cosh (J))-\lim _{N \rightarrow \infty} \frac{2 \ln Z(J)}{N} .
\end{aligned}
$$

Let us denote the upper bound in 126 by $U(J)$, which is plotted by the dashed-dotted red line in Fig. 17. The derivative of $U(J)$ with respect to the coupling parameter $J$ is

$$
\frac{\partial U(J)}{\partial J}=2+2 \tanh (J)-\lim _{N \rightarrow \infty} \frac{2}{N} \frac{\partial \ln Z(J)}{\partial J} .
$$

From 97, it is straightforward to verify that 127 is zero at the critical coupling $J_{\mathrm{c}}$ given by 96 . From Fig. 17 we observe that the upper bound $U(J)$ grows to attain its maximum at $J_{\mathrm{c}}$, but then decays for $J>J_{\mathrm{c}}$. (Again, recall from Section $\mathrm{V}-\mathrm{C}$ that (48) vanishes as $J \rightarrow \infty$.)

\section{ACKNOWLEDGMENTS}

The authors are grateful to Hans-Andrea Loeliger for his comments and discussions related to the topics of this paper. The authors would like to thank David Forney, Pascal Vontobel, Justin Dauwels, Alistair Sinclair, and Stefan Moser for their helpful comments on an earlier draft of this manuscript. The authors wish to thank the associate editor, Yongyi Mao, for his constructive suggestions during the review process. Part of this work was done during the first author's stay at the Institut Henri Poincaré - Centre Emile Borel, France, at the Information Theory and Coding Group, University of Pompeu Fabra, Spain, and at the Department of Statistics and Actuarial Science, University of Waterloo, Canada. The first author thanks these institutions for hospitality and support. This work is supported in part by the Spanish Ministry of Economy and Competitiveness under the Mara de Maeztu Units of Excellence Programme (MDM-2015-0502) and the Ramon y Cajal program RYC-2015-18878 (AEI/MINEICO/FSE,UE).

\section{REFERENCES}

[1] M. Molkaraie and H.-A. Loeliger, "Partition function of the Ising model via factor graph duality," Proc. 2013 IEEE Int. Symp. on Inf. Theory, Istanbul, Turkey, July 7-12, 2013, pp. 2304-2308.

[2] M. Molkaraie, "An importance sampling scheme for models in a strong external field,' Proc. 2015 IEEE Int. Symp. on Inf. Theory, Hong Kong, June 14-19, 2015, pp. 1179-1183.

[3] M. Molkaraie, "An importance sampling algorithm for the Ising model with strong couplings," Proc. 2016 Int. Zurich Seminar on Communications (IZS), Zurich, Switzerland, March 2-4, 2016, pp. 180-184.

[4] F. R. Kschischang, B. J. Frey, and H.-A. Loeliger, "Factor graphs and the sum-product algorithm," IEEE Trans. Inf. Theory, vol. 47, pp. 498-519, Feb. 2001.

[5] H.-A. Loeliger, "An introduction to factor graphs," IEEE Signal Proc. Mag., vol. 29, pp. 28-41, Jan. 2004.

[6] S. M. Aji and R. J. McEliece, "The generalized distributive law," IEEE Trans. Inf. Theory, vol. 46, pp. 325-343, March 2000.

[7] R. J. Baxter, Exactly Solved Models in Statistical Mechanics. Dover Publications, 2007.

[8] J. M. Yeomans, Statistical Mechanics of Phase Transitions. Oxford University Press, 1992.

[9] J. S. Yedidia, W. T. Freeman, and Y. Weiss, "Constructing free-energy approximations and generalized belief propagation algorithms," IEEE Trans. Inf. Theory, vol. 51, pp. 2282-2312, 2005.

[10] Y. Qi and T. P. Minka, "Tree-structured approximations by expectation propagation," Advances in Neural Information Processing Systems (NIPS), Dec. 2004, pp. 193-200.

[11] E. Ising, "Beitrag zur theorie des ferromagnetismus," Zeitschrift für Physik A Hadrons and Nuclei, vol. 31, pp. 253-258, Feb. 1925.

[12] B. A. Cipra, "An introduction to the Ising model," American Mathematical Monthly, vol. 94, pp. 937-959, Dec. 1987.

[13] R. B. Potts, "Some generalized order-disorder transformations," Proc. the Cambridge Philosophical Society, vol. 48, pp. 106-109, Jan. 1952.

[14] F. Y. Wu, "The Potts model," Rev. of Modern Phys., vol. 54, pp. 235 268, Jan. 1982.

[15] Y. Boykov, O. Veksler, and R. Zabih, "Fast approximate energy minimization via graph cuts," IEEE Trans. on Pattern Analysis and Machine Intelligence, vol. 23, pp. 1222-1239, Nov. 2001. 
[16] J. Besag and P. J. Green, "Spatial statistics and Bayesian computation," Journal of the Royal Statistical Society. Series B, vol. 55, pp. 25-37, 1993

[17] F. Y. Wu, "Potts model and graph theory," Journal of Statistical Physics, vol. 52, pp. 99-112, July 1988.

[18] A. D. Sokal, "Bounds on the complex zeros of (di) chromatic polynomials and Potts-model partition functions," Combinatorics, Probability and Computing, vol. 10, pp. 41-77, 2001.

[19] A. D. Sokal, "The multivariate Tutte polynomial (alias Potts model) for graphs and matroids," Surveys in Combinatorics, vol. 327, pp. 173-226, Cambridge University Press, 2005.

[20] P. W. Kasteleyn, "Dimer statistics and phase transitions," Journal of Mathematical Physics, vol. 4, pp. 287-293, Feb. 1963.

[21] M. E. Fisher, "On the dimer solution of planar Ising models," Journal of Mathematical Physics, vol. 7, pp. 1776-1781, Oct. 1966

[22] V. Gómez, H. J. Kappen, and M. Chertkov, "Approximate inference on planar graphs using loop calculus and belief propagation," Journal of Mach. Learn. Res., vol. 11, pp. 1273-1296, April 2010.

[23] V. V. Vazirani, Approximation Algorithms. Springer, 2004

[24] M. Jerrum and A. Sinclair, "Polynomial-time approximation algorithms for the Ising model," SIAM Journal on Computing, vol. 11, pp. 10871116, Oct. 1993.

[25] L. A. Goldberg and M. Jerrum, "Approximating the partition function of the ferromagnetic Potts model," Journal of the ACM, vol. 59, pp. 12221239, Oct. 2012.

[26] L. A. Goldberg and M. Jerrum, "The complexity of computing the sign of the Tutte polynomial," SIAM Journal on Computing, vol. 43, pp. 1921-1952, Dec. 2014.

[27] A. Galanis, D. Štefankovič, E. Vigoda, and L. Yang, "Ferromagnetic Potts model: refined \#BIS-hardness and related results," SIAM Journal on Computing, vol. 45, pp. 2004-20065, Nov. 2016.

[28] J. M. Hammersley and D. C. Handscomb, Monte Carlo Methods. Methuen \& Co., London, 1964.

[29] R. M. Neal, Probabilistic Inference Using Markov Chain Monte Carlo Methods. Techn. Report CRG-TR-93-1, Dept. Computer Science, Univ. of Toronto, Sept. 1993.

[30] K. Binder and D. W. Heermann, Monte Carlo Simulation in Statistical Physics. Springer, 2010

[31] M. Molkaraie and H.-A. Loeliger, "Monte Carlo algorithms for the partition function and information rates of two-dimensional channels," IEEE Trans. Inf. Theory, vol. 59, pp. 495-503, Jan. 2013.

[32] G. Potamianos and J. Goutsias, "Stochastic approximation algorithms for partition function estimation of Gibbs random fields," IEEE Trans. Inf. Theory, vol. 43, pp. 1984-1965, Nov. 1997.

[33] I. Murray, D. Mackay, Z. Ghahramani, and J. Skilling, "Nested sampling for Potts models," Advances in Neural Information Processing Systems (NIPS), Dec. 2005, pp. 947-954.

[34] R. H. Swendsen and J. S. Wang, "Nonuniversal critical dynamics in Monte Carlo simulations," Phys. Rev. Letters, vol. 58, pp. 86-88, Jan. 1987.

[35] G. D. Forney, Jr., "Codes on graphs: normal realizations," IEEE Trans. Inf. Theory, vol. 47, pp. 520-548, Feb. 2001.

[36] A. Al-Bashabsheh and Y. Mao, "Normal factor graphs and holographic transformations," IEEE Trans. Inf. Theory, vol. 57, pp. 752-763, Feb. 2011.

[37] G. D. Forney, Jr., "Codes on graphs: duality and MacWilliams identities," IEEE Trans. Inf. Theory, vol. 57, pp. 1382-1397, Feb. 2011.

[38] G. D. Forney, Jr. and P. O. Vontobel, "Partition functions of normal factor graphs," 2011 Information Theory and Applications Workshop, La Jolla, USA, Feb. 6-11, 2011.

[39] H. A. Kramers and G. H. Wannier, "Statistics of the two-dimensional ferromagnet. Part I,” Phys. Rev., vol. 60, pp. 252-262, Aug. 1941.
[40] A. Al-Bashabsheh and P. Vontobel, "The Ising model: Kramers-Wannier duality and normal factor graphs," Proc. 2015 IEEE Int. Symp. on Inf. Theory, Hong Kong, June 14-19, 2015, pp. 2266-2270.

[41] A. Al-Bashabsheh and P. Vontobel, "A factor-graph approach to algebraic topology, with applications to Kramers-Wannier duality," IEEE Trans. Inf. Theory, vol. 64, pp. 7488-7510, Dec. 2018.

[42] G. D. Forney, Jr., "Codes on graphs: Models for elementary algebraic topology and statistical physics," IEEE Trans. Inf. Theory, vol. 64, pp. 7465-7487, Dec. 2018.

[43] A. Al-Bashabsheh and Y. Mao, "On stochastic estimation of the partition function," Proc. 2014 IEEE Int. Symp. on Inf. Theory, Honolulu, USA, June 29 - July 4, 2014, pp. 1504-1508.

[44] L. Onsager, "Crystal statistics. I. A two-dimensional model with an order-disorder transition," Phys. Rev., vol. 65, pp. 117-149, Feb. 1944.

[45] M. Molkaraie, "The primal versus the dual Ising model," Proc. 55th Annual Allerton Conf. on Communication, Control, and Computing, Monticello, USA, Oct. 3-6, 2017, pp. 53-60.

[46] B. Bollobás, Modern Graph Theory. Springer, 1998.

[47] I. Csiszár and P. C. Shields, "Information theory and statistics: a tutorial," Foundations and Trends in Communications and Information Theory, vol. 1, 2004, pp. 417-528.

[48] T. H. Cormen, C. E. Leiserson, C. Eric, R. L. Rivest, and C. Stein, Introduction to Algorithms. MIT Press, 2009.

[49] K. P. Murphy, Machine Learning: A Probabilistic Perspective. MIT Press, 2012

[50] J. S. Yedidia, J. S. Freeman, W. T. Freeman, and Y. Weiss, "Generalized belief propagation," Advances in Neural Information Processing Systems (NIPS), Nov. 2000, pp. 689-695.

[51] T. Heskes, A. Kees, and B. Kappen, "Approximate inference and constrained optimization," Uncertainty in Artificial Intelligence (UAI), Aug. 2002, pp. 313-320.

[52] J. M. Mooij, "libDAI: A free and open source C++ library for discrete approximate inference in graphical models," Journal of Mach. Learn. Res., vol. 11, pp. 2169-2173, Aug. 2010.

[53] Y. Ogata and M. Tanemura, "Estimation of interaction potentials of spatial point patterns through the maximum likelihood procedure," Ann. Inst. Statist. Math., vol. 33, pp. 315-338, 1981.

[54] R. M. Neal, “Annealed importance sampling," Statistics and Computing, vol. 11, pp. 125-139, April 2001.

[55] H. Nishimoro and G. Oritz, Elements of Phase Transition and Critical Phenomena. Oxford University Press, 2011.

[56] R. J. McEliece, The Theory of Information and Coding: A Mathematical Framework for Communication. Addison-Wesley, 1977.

[57] G. F. Newell and E. W. Montroll, "On the theory of the Ising model of ferromagnetism," Rev. of Modern Phys., vol. 25, pp. 353-389, April 1953.

[58] M. Molkaraie and H.-A. Loeliger, "Extending Monte Carlo methods to factor graphs with negative and complex factors," Proc. 2012 IEEE Information Theory Workshop, Lausanne, Switzerland, Sept. 3-7, 2012, pp. 367-371.

[59] M. X. Cao and P. O. Vontobel, "Estimating the information rate of a channel with classical input and output and a quantum state," Proc. 2017 IEEE Int. Symp. on Inf. Theory, Aachen, Germany, June 25-30, 2017 pp. 3205-3209.

[60] M. X. Cao and P. O. Vontobel, "Double-edge factor graphs: definition, properties, and examples," Proc. 2017 IEEE Information Theory Workshop, Kaohsiung, Taiwan, Nov. 6-10, 2017, pp. 136-140.

[61] H.-A. Loeliger and P. O. Vontobel, "Factor graphs for quantum probabilities," IEEE Trans. Inf. Theory, vol. 63, pp. 5642-5665, Sept. 2017.

[62] B. M. McCoy and T. T. Wu, The Two-Dimensional Ising Model. Courier Corporation, 2014. 